\title{
A class of weighted projective curves arising in representation theory of finite dimensional algebras
}

\author{
Werner Geigle and Helmut Lenzing \\ Fachbereich Mathematik-Informatik \\ Universität-GH Paderborn \\ Warburger Str. 100 \\ D-4790 Paderborn \\ West-Germany
}

\section{Introduction}

By means of a suitably graded sheaf theory we introduce a new class of curves, called weighted projective lines, having an interpretation as lines in an appropriate weighted projective space $\mathbf{P}_{n}(\boldsymbol{p})$, with respect to a weight sequence $\boldsymbol{p}=\left(p_{0}, \ldots, p_{n}\right)$ of integers. We note that our approach to weighted projective spaces is similar to the treatment by Delorme [8], Dolgachev [10] and Beltrametti-Robbiano [6] but differs sensibly in spirit and content. Section 1 summarizes those results of joint investigation with D. Baer and P. Dowbor which are needed to put weighted projective lines into proper perspective; a complete account is under preparation. The main advantage of our approach is that Serre's theorem (1.7) holds true, which removes all the pathologies ( [6], Section 3) encountered in the former treatment of these spaces.

As becomes clear from the results of Sections 2 and 5, a weighted projective line $C$ behaves like a smooth projective curve with respect to coherent sheaves and vector bundles on $\boldsymbol{C}$. This allows us to use all the methods familiar in this latter situation, see [24], [32], [1]. So the category of coherent sheaves $\operatorname{coh}(\boldsymbol{C})$ has Serre-duality (2.2), consequently almost-split sequences (2.3). Each coherent sheaf splits into a direct sum of a vector bundle and a torsion sheaf (2.4). By means of a Riemann-Roch theorem (2.9) we 
attach a (virtual) genus to $\boldsymbol{C}$, which is characteristic for the complexity of the classification problem for $\operatorname{coh}(\boldsymbol{C})(5.4)$.

Our motivation to investigate weighted projective lines originates from the representation theory of finite dimensional algebras in an attempt to give a geometric treatment similar to [26] for the so-called canonical algebras, introduced and studied by C. M. Ringel [30]. Actually there is a bijective correspondence between (isomorphism classes of) weighted projective lines and canonical algebras, respectively.

The reader will observe that our present treatment differs sensibly from the previous approach, using a variant of Beilinson's theorem [5] as the basis for the comparison between $\operatorname{coh}(C)$ and $\bmod (\Lambda)$ : By means of a tilting sheaf (3.1) with endomorphism algebra $\Lambda$, we establish an equivalence $\mathrm{D}^{b}(\operatorname{coh}(\boldsymbol{C}))=\mathrm{D}^{b}\left(\bmod \left(\Lambda^{o p}\right)\right)$ of the derived categories of $\operatorname{coh}(\boldsymbol{C})$ and the category $\bmod \left(\Lambda^{o p}\right)$ of finite dimensional $\Lambda$-modules, respectively. A comparison theorem (3.3) deduces the consequences of $\mathrm{D}^{b}(\operatorname{coh}(\boldsymbol{C}))=\mathrm{D}^{b}\left(\bmod \left(\Lambda^{o p}\right)\right)$ in the spirit of tilting theory [20], [7], [21]. As a result, the classification problems for $\operatorname{coh}(\boldsymbol{C})$ and $\bmod (\Lambda)$, if $\Lambda$ denotes the canonical algebra attached to $\boldsymbol{C}$, are basically equivalent. We note that the subdivision of indecomposable coherent sheaves on $\boldsymbol{C}$ into the two classes of indecomposable vector bundles and torsion sheaves translates by means of tilting into a subdivision of indecomposable $\Lambda$-modules into now three classes (cf. (4.3) and [30]).

In Section 5 we give a brief account on the classification of indecomposable bundles on $C$ if $\boldsymbol{C}$ has (virtual) genus one. Not unexpectedly, Atiyah's approach to classify vector bundles on smooth elliptic projective curves [1] also works in this context. Our exposition also relies on the work of Narasimhan and Seshadri (see [32]). Thus the comparison $\mathrm{D}^{b}(\operatorname{coh}(\boldsymbol{C}))=\mathrm{D}^{b}\left(\bmod \left(\Lambda^{o p}\right)\right)$ establishes a link between Atiyah's classification of vector bundles on elliptic curves and Ringel's classification for modules over canonical algebras of tubular type [30]. From a geometric point of view this interrelation is explained by Example 5.8.

Theorem 5.1 relates the classification of vector bundles on $\boldsymbol{C}$ to the study of graded Cohen-Macaulay modules; a detailed account will be given elsewhere.

The authors acknowledge the support of the Deutsche Forschungsgemeinschaft (SPP Darstellungstheorie). The second-named author wishes to thank C. S. Seshadri for helpful discussions pointing out the interrelations of weighted projective lines to the concept of curves with parabolic structure [32]. 


\section{Weighted projective spaces and weighted projective lines}

Throughout $k$ denotes an algebraically closed field of arbitrary characteristic.

1.1. Let $\boldsymbol{p}=\left(p_{0}, \ldots, p_{n}\right)$ be an $(n+1)$-tuple of integers $p_{i} \geq 1$, called the weight sequence. The affine algebraic group

$$
G(p)=\left\{\left(t_{0}, t_{1}, \ldots, t_{n}\right) \in\left(k^{*}\right)^{n+1} \mid t_{0}^{p_{0}}=t_{1}^{p_{1}}=\cdots=t_{n}^{p_{n}}\right\}
$$

acts on affine $(n+1)$-space $\mathbf{A}_{n+1}=k^{n+1}$ by multiplication

$$
\left(t_{0}, t_{1}, \ldots, t_{n}\right)\left(x_{0}, x_{1}, \ldots, x_{n}\right)=\left(t_{0} x_{0}, \ldots, t_{n} x_{n}\right) .
$$

If $\mathbf{L}(p)$ denotes the rank one abelian group on generators $\vec{x}_{0}, \vec{x}_{1}, \ldots, \vec{x}_{n}$ with relations $p_{0} \vec{x}_{0}=p_{1} \vec{x}_{1}=\cdots=p_{n} \vec{x}_{n}$, clearly the group Hopf algebra $k[\mathbf{L}(\boldsymbol{p})]$ of $\mathbf{L}(\boldsymbol{p})$ represents the affine algebraic group $G(p)$. Hence the above $G(\boldsymbol{p})$-action on $\mathbf{A}_{n+1}$ corresponds to a graduation of the polynomial algebra $S=k\left[X_{0}, X_{1}, \ldots, X_{n}\right]$ with grading $\operatorname{group} \mathbf{L}(\boldsymbol{p})$; the graduation being specified by defining $X_{i}$ to be homogeneous of degree $\vec{x}_{i}$. (Notation: $\operatorname{deg}\left(X_{i}\right)=\vec{x}_{i}$ ). Thus $S$ carries a decomposition $S=\emptyset S_{\vec{l}}$ into $k$-subspaces satisfying $\vec{l} \in \mathbf{L}(p)$

$S_{\vec{l}} S_{\vec{m}} \subset S_{\vec{l}+\vec{m}}$ for all $\vec{l}, \vec{m} \in \mathbf{L}(\boldsymbol{p})$; moreover $X_{i} \in S_{\vec{x}_{i}}$ for $i=0, \ldots, n$. We use the notation $S(\boldsymbol{p})$ for the $\mathbf{L}(\boldsymbol{p})$-graded algebra thus defined.

To each sequence $\lambda=\left(\lambda_{0}, \ldots, \lambda_{n}\right)$ of pairwise distinct elements of $\mathbf{P}_{1}(k)$, normalized such that $\lambda_{0}=\infty, \lambda_{1}=0, \lambda_{2}=1$, we attach the two-dimensional subvariety $\boldsymbol{F}(\boldsymbol{p}, \lambda)$ of $\mathbf{A}_{n+1}$, given by the equations

$$
X_{i}^{p_{i}}=X_{1}^{p_{1}}-\lambda_{i} X_{0}^{p_{0}}, \quad i=2, \ldots, n .
$$

$\boldsymbol{F}(\boldsymbol{p}, \boldsymbol{\lambda})$ is stable under the $G(\boldsymbol{p})$-action just described. Accordingly, the elements $f_{i}=X_{i}^{p_{i}}-X_{1}^{p_{1}}+\lambda_{i} X_{0}^{p_{0}}, \quad(i=2, \ldots, n)$ generate a homogeneous ideal $I(\boldsymbol{p}, \lambda)$ of $S(p)$. Hence

$$
S(\boldsymbol{p}, \lambda)=k\left[X_{0}, X_{1}, \ldots, X_{n}\right] / I(\boldsymbol{p}, \lambda)=k\left[x_{0}, x_{1}, \ldots, x_{n}\right]
$$

is again $\mathbf{L}(\boldsymbol{p})$-graded with $\operatorname{deg}\left(x_{i}\right)=\vec{x}_{i}$.

We are now going to endow the (set-theoretic) quotients $\mathbf{P}_{n}(p)=k^{n+1}-\{0\} / G(\boldsymbol{p})$ and $\boldsymbol{C}(\boldsymbol{p}, \boldsymbol{\lambda})=\boldsymbol{F}(\boldsymbol{p}, \boldsymbol{\lambda}) / G(\boldsymbol{p})$ with an $\mathbf{L}(\boldsymbol{p})$-graded sheaf theory, defining on $\mathbf{P}_{n}(\boldsymbol{p})$ and $C(p, \lambda)$ the geometric structure of a weighted projective space, a weighted projective line, respectively. 
1.2. Call $\vec{c}=p_{0} \vec{x}_{0}=\cdots=p_{n} \vec{x}_{n}$ the canonical element of $\mathbf{L}(\boldsymbol{p})$. For reasons, which will become clear later, $\vec{\omega}=(n-1) \vec{c}-\sum_{i=0}^{n} \vec{x}_{i}$ is called the dualizing element. $\mathbf{L}(\boldsymbol{p})$ is an ordered group with $L_{+}=\sum_{i=0}^{n} \mathbf{N} \vec{x}_{i}$ as its set of positive elements. Since $\mathbf{L}(\boldsymbol{p}) / \mathbf{Z} \vec{c} \tilde{=} \prod_{i=0}^{n} \mathbf{Z} / p_{i} \mathbf{Z}$ canonically, each $\vec{l} \in \mathbf{L}(\boldsymbol{p})$ can be uniquely written in normal form

$$
\vec{l}=\sum_{i=0}^{n} l_{i} \vec{x}_{i}+l \vec{c} \text { with } 0 \leq l_{i}<p_{i} \text { and } l \in \mathbf{Z} .
$$

If $\vec{l}, \vec{m}$ are both in normal form, $\vec{l} \leq \vec{m}$ if and only if $l_{i} \leq m_{i}$ for $i=0, \ldots, n$ and $l \leq m$.

Since $\vec{c}+\vec{\omega}=\sum_{i=0}^{n}\left(p_{i}-1\right) \vec{x}_{i}-\vec{c}$ is in normal form, we see that each $\vec{l} \in \mathbf{L}(\boldsymbol{p})$ satisfies exactly one of the two possibilities: $0 \leq \vec{l}$ or $\vec{l} \leq \vec{c}+\vec{\omega}$.

Let $p=1 . c . m . ~\left(p_{0}, \ldots, p_{n}\right)$. We define the degree map $\delta: \mathbf{L}(\boldsymbol{p})-\rightarrow \mathbf{Z}$ on generators by $\delta\left(\vec{x}_{i}\right)=\frac{p}{p_{i}} . \delta$ is an epimorphism of ordered groups, its kernel being the torsion group of $\mathbf{L}(\boldsymbol{p})$. Note that $\delta(\vec{l})=0$ implies $p \vec{l}=0$.

For an $\mathbf{L}(\boldsymbol{p})$-graded algebra $\operatorname{Mod}^{\mathbf{L}(p)}(S), \bmod ^{\mathbf{L}(p)}(S)$ and $\bmod _{0} \mathbf{L}(p)(S)$ denote the categories of all (all finitely generated, all finite length) $\mathbf{L}(\boldsymbol{p})$-graded $S$-modules, respectively, with morphisms the $S$-linear homogeneous maps of degree $0 . \mathbf{L}(\boldsymbol{p})$ acts on each of this categories by twist $(M, \vec{x})-\rightarrow M(\vec{x})$, where $M(\vec{x})_{\vec{y}}=M_{\vec{x}+\vec{y}}$. Similar notations are used for ungraded, also for $\mathbf{L}_{+}$-graded modules.

1.3. For the applications we have in mind, the following proposition serves as the basic tool:

Proposition. $S(\boldsymbol{p})$ and $S(\boldsymbol{p}, \lambda)$ are $\mathbf{L}(\boldsymbol{p})$-graded factorial domains, $i$. e. up to scalars each homogeneous element is a product of homogeneous prime elements. Up to scalars a complete system of homogeneous prime elements for $S(\boldsymbol{p})$ or $S(\boldsymbol{p}, \lambda)$ is given by

(i) the elements $X_{0}, X_{1}, \ldots, X_{n} \in S(\boldsymbol{p}),\left(x_{0}, x_{1}, \ldots, x_{n} \in S(\boldsymbol{p}, \lambda)\right)$, called the exceptional prime elements of $S(\boldsymbol{p}), S(\boldsymbol{p}, \lambda)$, respectively.

(ii) the elements $f\left(X_{0}^{p_{0}}, \ldots, X_{n}^{p_{n}}\right),\left(f\left(x_{0}^{p_{0}}, x_{1}^{p_{1}}\right)\right)$, called the ordinary prime elements, where $f$ is an irreducible homogeneous element of the polynomial algebra $k\left[T_{0}, T_{1}, \ldots, T_{n}\right],\left(k\left[T_{0}, T_{1}\right]\right)$, not associated to any $T_{0}, T_{1}, \ldots, T_{n},\left(T_{0}, T_{1}\right)$, respectively. Here, as usual, both polynomial algebras are $\mathbf{Z}$-graded by total degree. 
Moreover, $x_{0}, x_{1}, \ldots, x_{n},\left(x_{0}, x_{1}\right)$ is an $\mathbf{L}(\boldsymbol{p})$-homogeneous regular sequence for $S(\boldsymbol{p})$ or $S(p, \lambda)$, respectively. In particular, $S(\boldsymbol{p}), S(\boldsymbol{p}, \lambda)$ has graded and ungraded Krull dimension $n+1$ or 2 , respectively. Also $x_{0}, x_{1}, f_{2}, \ldots, f_{n}$ is a regular sequence for $S(\boldsymbol{p})$.

Since we assume that $k$ is algebraically closed, the elements

$$
x_{1}^{p_{1}}-\lambda x_{0}^{p_{0}}, \lambda \in k-\left\{\lambda_{0}, \ldots, \lambda_{n}\right\}
$$

constitute a complete set of ordinary primes for $S(\boldsymbol{p}, \lambda)$.

Proof. Let first $S$ denote the algebra $S(\boldsymbol{p})$. We may view the restriction

$$
R(p)=\bigoplus_{l=0}^{\infty} R_{l}, \quad R_{l}=S_{l \vec{c}}
$$

of $S$ to the subgroup $\mathbf{Z} \vec{c}$ of $\mathbf{L}(p)$ as a $\mathbf{Z}$-graded algebra, called the core of $S$. Clearly, $R(\boldsymbol{p})=k\left[X_{0}^{p_{0}}, \ldots, X_{n}^{p_{n}}\right]$ is the $\mathbf{Z}$-graded polynomial algebra over $k$ in the indeterminates $X_{0}^{p_{0}}, \ldots, X_{n}^{p_{n}}$, and $R_{l}$ consists of all homogeneous polynomials of total degree $l$ in $X_{0}^{p_{0}}, \ldots, X_{n}^{p_{n}}$. Moreover if $\vec{l} \in \mathbf{L}(\boldsymbol{p})$ is written in normal form (1.2.1) we have

$$
S(\boldsymbol{p})_{\vec{l}}=X_{0}^{l_{0}} \cdots X_{n}^{l_{n}} R(\boldsymbol{p})_{l}, \text { where } 0 \leq l_{i}<p_{i}, l \in \mathbf{Z} .
$$

Similarly, the core of $S(\boldsymbol{p}, \boldsymbol{\lambda})$ is the polynomial algebra $R(\boldsymbol{p}, \boldsymbol{\lambda})=k\left[x_{0}^{p_{0}}, x_{1}^{p_{1}}\right]$ and

$$
S(\boldsymbol{p}, \lambda)_{\vec{l}}=x_{0}^{l_{0}} \cdots x_{n}^{l_{n}} R(\boldsymbol{p}, \lambda)_{l}, \quad \text { where } 0 \leq l_{i}<p_{i}, \quad l \in \mathbf{Z} .
$$

The assertions now follow from (1.3.2) and (1.3.3).

As is clear from (1.3.2) and (1.3.3) we have $S_{\vec{l}} \neq 0$ if and only if $\vec{l} \geq 0$.

The role of the algebras $S(\boldsymbol{p}, \lambda)$, with $p_{0}, \ldots, p_{n}$ pairwise coprime, is explained by a theorem of Mori [27], stating that for an algebraically closed base field $k$ these are just the Z-graded affine $k$-algebras $S$ with $S_{0}=k$, which are graded factorial of Krull dimension two.

1.4. Let $S$ be either $S(\boldsymbol{p})$ or $S(\boldsymbol{p}, \lambda)$ and $R$ be the core of $S$. If $T$ is a multiplicative subset of $R$, consisting of homogeneous elements, the quotient ring $T^{-1} S$ is again $\mathbf{L}(\boldsymbol{p})$-graded: $\left(T^{-1} S\right)_{\vec{l}}$ consists of all homogeneous quotients $\frac{s}{t}$, where $s \in S, t \in T$ both are homogeneous and $\operatorname{deg}(s)-\operatorname{deg}(t)=\vec{l}$. Replacing $T$ by $T^{p}=\left\{t^{p} \mid t \in T\right\}$, if necessary, we may always assume that $T$ is contained in $R$. In this case, for $\vec{l} \in \mathbf{L}(\boldsymbol{p})$ written in normal form (1.2.1) we get

$$
\left(T^{-1} S\right)_{\vec{l}}=X_{0}^{l_{0}} \cdots X_{n}^{l_{n}}\left(T^{-1} R\right)_{l}
$$


If $T$ is the set of all homogeneous non-zero elements of $R, Q=T^{-1} S$ is the total ring of homogeneous quotients. Its zero-component $Q_{0}$ is the field of rational functions $k\left(\frac{X_{1}^{p_{1}}}{X_{0}^{p_{0}}}, \ldots, \frac{X_{n}^{p_{n}}}{X_{0}^{p_{0}}}\right)$ or $k\left(\frac{x_{1}^{p_{1}}}{x_{0}^{p_{0}}}\right)$, according as $S=S(\boldsymbol{p})$ or $S=S(\boldsymbol{p}, \boldsymbol{\lambda})$. Moreover, passage to the zero-component

$$
\bmod ^{\mathbf{L}(p)}(Q)-\rightarrow \bmod \left(Q_{0}\right), M-\rightarrow M_{0}
$$

defines a category equivalence. We express this fact, stating that the $\mathbf{L}(\boldsymbol{p})$-graded algebra $Q$ is Morita-equivalent to the algebra $Q_{0}$.

1.5. Let $\boldsymbol{X}$ be either $\mathbf{P}_{n}(\boldsymbol{p})$ or its subset $\boldsymbol{C}(\boldsymbol{p}, \lambda)$, accordingly $S$ stands either for $S(\boldsymbol{p})$ or $S(\boldsymbol{p}, \lambda)$. For $\vec{l}=l_{0} \vec{x}_{0}+\cdots+l_{n} \vec{x}_{n}$ and $t=\left(t_{0}, t_{1}, \ldots, t_{n}\right) \in G(\boldsymbol{p})$ we write

$$
\vec{l}(t)=t_{0}^{p_{0}} \cdots t_{n}^{p_{n}},
$$

which identifies $\vec{l}$ with a character of $G(\boldsymbol{p})$, actually $\mathbf{L}(\boldsymbol{p})$ with $\operatorname{Hom}\left(G(\boldsymbol{p}), k^{*}\right)$. We have

$$
f(t x)=\vec{l}(t) f(x) \text { for } f \in S_{\vec{l}}, x \in k^{n+1}, t \in G(\boldsymbol{p}) .
$$

This allows to form the sets

$$
\mathrm{D}(f)=\{[x] \in \boldsymbol{X} \mid f(x)=0\}, f \in S \text { homogeneous , }
$$

which form a basis for the Zariski topology on $\boldsymbol{X}$. As usual $\mathrm{V}(f)$ denotes the complement of $\mathrm{D}(f)$ in $\boldsymbol{X}$. The structure sheaf $\boldsymbol{O}_{\boldsymbol{X}}$ is the sheaf of $\mathbf{L}(\boldsymbol{p})$-graded $k$-algebras attached to the presheaf $\mathrm{D}(f) \rightarrow S_{f}, f \in S$ homogeneous. $S_{f}$ denotes the $\mathbf{L}(\boldsymbol{p})$-graded quotient ring $T^{-1} S$, where $T$ is the multiplicative set generated by $f$. As usual ( [17]), we have

$$
\Gamma\left(\mathrm{D}(f), \boldsymbol{O}_{\boldsymbol{X}}\right)=S_{f}
$$

if $f$ is a homogeneous element of $S_{+}=\underset{\vec{l}>0}{\bigoplus} S_{\vec{l}}$. By graded normality of $S$ (cf. Proposition 1.3), we also get

$$
\Gamma\left(X, \boldsymbol{O}_{\boldsymbol{X}}(\vec{x})\right)=S(\vec{x}) \text { for each } \vec{x} \in \mathbf{L}(\boldsymbol{p}) .
$$

As a result, $\boldsymbol{O}_{\boldsymbol{X}}$ is an $\mathbf{L}(\boldsymbol{p})$-graded sheaf of algebras

$$
\boldsymbol{O}_{\boldsymbol{X}}=\bigoplus_{\vec{l} \in \mathbf{L}(p)}\left(\boldsymbol{O}_{\boldsymbol{X}}\right)_{\vec{l}}
$$

We note that our approach differs sensibly from the traditional treatment of weighted projective spaces [ 8], [10], [6] where the 0-component $\left(\boldsymbol{O}_{\boldsymbol{X}}\right)_{0}$ of $\boldsymbol{O}_{P_{n}(p)}$ serves as the structure sheaf. (In order to make this comparison possible, we have to assume that $p_{0}, \ldots, p_{n}$ are pairwise coprime, so $\mathbf{L}(\boldsymbol{p})=\mathbf{Z}$.). 
The weight $\boldsymbol{p}(t)$ of a point $t=\left[t_{0}, \ldots, t_{n}\right]$ is defined as $\Pi\left\{p_{j} / t_{j}=0\right\}$. A point $t$ is called ordinary if $p(t)=1$, otherwise $t$ is called exceptional. It follows from Proposition 1.3 that we may view $\boldsymbol{C}(\boldsymbol{p}, \lambda)$ as a curve in $\mathbf{P}_{n}(\boldsymbol{p})$, which is a complete intersection since the defining equations $f_{i}=X_{i}^{p_{i}}-X_{1}^{p_{1}}+\lambda_{i} X_{0}^{p_{0}}$ form a regular sequence of homogeneous elements in $S(\boldsymbol{p})$. Actually we prefer to call $\boldsymbol{C}(\boldsymbol{p}, \lambda)$ a weighted projective line in $\mathbf{P}_{n}(\boldsymbol{p})$ : the map

$$
\mathbf{P}_{n}(\boldsymbol{p})-\rightarrow \mathbf{P}_{n}(k),\left[x_{0}, x_{1}, \ldots, x_{n}\right]-\rightarrow\left[x_{0}^{p_{0}}, \ldots, x_{n}^{p_{n}}\right]
$$

allows to identify both spaces set-theoretically. By means of (1.5.7) the defining equations for $\boldsymbol{C}(\boldsymbol{p}, \boldsymbol{\lambda})$ convert into equations defining a line in $\boldsymbol{P}_{n}(k)$. Accordingly

$$
\boldsymbol{C}(\boldsymbol{p}, \boldsymbol{\lambda})-\rightarrow \boldsymbol{P}_{1}(k), \quad\left[x_{0}, x_{1}, \ldots, x_{n}\right]-\rightarrow\left[x_{0}^{p_{0}}, x_{1}^{p_{1}}\right]
$$

is a bijection. By means of this correspondence the exceptional points of $\boldsymbol{C}(\boldsymbol{p}, \lambda)$ are just converted to the system $\lambda_{0}, \ldots, \lambda_{n}$, attaching weight $p_{i}$ to $\lambda_{i}$.

1.6. The stalk $\boldsymbol{O}_{X, t}$ of the structure sheaf $\boldsymbol{O}_{X}$ at $t \in \boldsymbol{X}$ is given by

$$
\boldsymbol{O}_{\boldsymbol{X}, t}=\left\{\frac{f}{g} \mid f, g \in S, g \text { homogeneous with } g(t) \neq 0\right\} \text {. }
$$

This ring is always $\mathbf{L}(\boldsymbol{p})$-graded regular local of dimension $n+1$ if $\boldsymbol{X}=\mathbf{P}_{n}(\boldsymbol{p})$, and an $\mathbf{L}(\boldsymbol{p})$-graded discrete valuation ring if $\boldsymbol{X}=\boldsymbol{C}(\boldsymbol{p}, \lambda)$. Notice that $R$ is called a graded local ring if $R$ has a unique homogeneous maximal ideal. Moreover regularity means that $R$ has finite graded global dimension $n$. If additionally $n=1$ we deal with a graded discrete valuation ring. For an ordinary point $t$, i. e. if all homogeneous coordinates $t_{i}$ are non-zero, $\boldsymbol{O}_{\boldsymbol{X}, t}$ is Morita-equivalent to its zero-component, which according to (1.4.1) is isomorphic to the (now ungraded) localization of the polynomial algebra $k\left[T_{1}, \ldots, T_{n}\right]$ with respect to the maximal ideal $\left(T_{1}-t_{1}, \ldots, T_{n}-t_{n}\right)$ or to the localization of $k[T]$ with respect to $\left(T-t_{1}\right)$ according as $\boldsymbol{X}=\mathbf{P}_{n}(\boldsymbol{p})$ or $\boldsymbol{X}=\boldsymbol{C}(\boldsymbol{p}, \lambda)$, respectively. Here, we assume $t_{0}=1$. Thus for an ordinary point, the category $\bmod ^{\mathbf{L}(p)}\left(\boldsymbol{O}_{\boldsymbol{X}, t}\right)$ of finitely generated $\mathbf{L}(\boldsymbol{p})$-graded modules over $\boldsymbol{O}_{\boldsymbol{X}, t}$ has exactly one simple module, up to isomorphism.

By contrast for an exceptional point $t$ of $\boldsymbol{X} \bmod ^{\mathbf{L}(\boldsymbol{p})}\left(\boldsymbol{O}_{X, t}\right)$ has exactly $\boldsymbol{p}(t)$ isomorphism classes of simple modules. Accordingly, $\bmod ^{\mathbf{L}(\boldsymbol{p})}\left(\boldsymbol{O}_{X, t}\right)$ has exactly $\boldsymbol{p}(t)$ isomorphism classes of indecomposable projective modules, necessarily of the form $\boldsymbol{O}_{\boldsymbol{X}, t}(\vec{x})$ for some $\vec{x} \in \mathbf{L}(p)$.

For $T$ an indeterminate let $A$ be the discrete valuation ring obtained from $k\left[T^{n}\right]$ by localizing at the maximal ideal $\left(T^{n}\right)$. It is easily checked from (1.4.1) that if $\boldsymbol{X}=\boldsymbol{C}(\boldsymbol{p}, \boldsymbol{\lambda})$ and $t$ denotes a point of weight $n$, the graded algebra $\boldsymbol{O}_{X, t}$ is Morita equivalent to the 
subring of $\mathrm{M}_{n}(k(T))$ given by

$$
\left[\begin{array}{cccccc}
A & T A & \cdot & \cdot & \cdot & T^{n-1} A \\
T^{n-1} A & A & & & \cdot \\
\cdot & \cdot & \cdot & & & \cdot \\
\cdot & & & \cdot & & \cdot \\
\cdot & & & A & T A \\
T A & \cdot & \cdot & \cdot & T^{n-1} A & A
\end{array}\right] .
$$

This proves that in the $\mathbf{L}(\boldsymbol{p})$-graded situation, we basically deal with a non-commutative sheaf theory.

We may attach a generic point $\xi$ to $\boldsymbol{X}$, where the stalk $\boldsymbol{F}_{\xi}=(\tilde{M})_{\xi}$ is given by $T^{-1} M$, with $T$ the set of all homogeneous non-zero divisors of $S$. As was shown in (1.4), $\boldsymbol{O}_{\xi}$ is Morita-equivalent to the function field $K$ of $\boldsymbol{X}$. Accordingly, we may view $\boldsymbol{F}_{\xi}$ as a (finite dimensional) vector space over $K$, whose dimension is called the rank of $\boldsymbol{F}$.

1.7. Again $\boldsymbol{X}$ stands for either $\mathbf{P}_{n}(\boldsymbol{p})$ or $\boldsymbol{C}(\boldsymbol{p}, \boldsymbol{\lambda})$. The sheaves on $\boldsymbol{X}$ we want to consider are the $\mathbf{L}(\boldsymbol{p})$-graded sheaves $\boldsymbol{M}$ of $\boldsymbol{O}_{\boldsymbol{X}}$-modules. Thus $\boldsymbol{M}$ is an $\boldsymbol{O}_{\boldsymbol{X}}$-module, carrying an $\mathbf{L}(\boldsymbol{p})$-graduation $\boldsymbol{M}=\underset{\vec{l} \in \mathbf{L}(\boldsymbol{p})}{\boldsymbol{M}_{\vec{l}}}$, satisfying $\boldsymbol{O}_{\boldsymbol{X}_{\vec{l}}} M_{\vec{m}} \subset M_{\vec{l}+\vec{m}}$. Morphisms will be morphisms of graded $\boldsymbol{O}_{X}$-modules of degree 0 . Let $\operatorname{Mod}^{\mathbf{L}(p)}\left(\boldsymbol{O}_{X}\right)$ be the resulting category of $\mathbf{L}(\boldsymbol{p})$-graded $\boldsymbol{O}_{X}$-modules. The group $\mathbf{L}(\boldsymbol{p})$ acts on $\operatorname{Mod}^{\mathbf{L}(\boldsymbol{p})}\left(\boldsymbol{O}_{X}\right)$ by twist

$$
(\vec{l}, \boldsymbol{M})-\rightarrow \boldsymbol{M}(\vec{l}),
$$

where $\boldsymbol{M}(\vec{l})_{\vec{x}}=\boldsymbol{M}_{\vec{l}+\vec{x}}$. Since $\boldsymbol{F}-\rightarrow \Gamma(\boldsymbol{X}, \boldsymbol{F})$ commutes with the shift operation, (1.5.5) generalizes to

$$
\Gamma\left(\boldsymbol{X}, \boldsymbol{O}_{X}(\vec{x})\right)=S(\vec{x}) \text { for each } \vec{x} \in \mathbf{L}(\boldsymbol{p}) .
$$

A coherent sheaf on $\boldsymbol{X}$ is by definition an $\mathbf{L}(\boldsymbol{p})$-graded $\boldsymbol{O}_{\boldsymbol{X}}$-module $\boldsymbol{M}$, where for each $x \in \boldsymbol{X}$ there is an open neighbourhood $U$ of $x$ and an exact sequence

$$
\bigoplus_{j=1}^{m} \boldsymbol{O}_{X}\left(\vec{l}_{j}\right)_{\left.\right|_{U}}-\rightarrow \bigoplus_{i=1}^{n} \boldsymbol{O}_{X}\left(\vec{l}_{i}\right)_{\left.\right|_{U}}-\rightarrow \boldsymbol{M}_{\left.\right|_{U}}-\rightarrow 0
$$

of $\mathbf{L}(\boldsymbol{p})$-graded $\boldsymbol{O}_{U}$-modules. $\operatorname{coh}(\boldsymbol{X})$ is the full subcategory of $\operatorname{Mod}^{\mathbf{L}(p)}\left(\boldsymbol{O}_{\boldsymbol{X}}\right)$ consisting of all coherent sheaves on $\boldsymbol{X}$. Quasicoherent sheaves are similarly defined, allowing infinite direct sums in (1.7.2). Qcoh $(\boldsymbol{X})$ denotes the category of all quasicoherent sheaves on $\boldsymbol{X}$. Both $\operatorname{coh}(\boldsymbol{X})$ and $\mathrm{Q} \operatorname{coh}(\boldsymbol{X})$ are stable under the twisting operation of $\mathbf{L}(\boldsymbol{p})$.

Given $\boldsymbol{M}, \boldsymbol{N} \in \operatorname{Mod}^{\mathbf{L}(\boldsymbol{p})}\left(\boldsymbol{O}_{X}\right)$, the presheaves of $\mathbf{L}(\boldsymbol{p})$-graded $\boldsymbol{O}_{X}$-modules given by 


$$
\begin{gathered}
U-\rightarrow \boldsymbol{M}(U) \boldsymbol{\sigma}_{X}(U) \\
\boldsymbol{N}(U), \\
U-\rightarrow \operatorname{HOM}_{\boldsymbol{O}_{X}(U)}(\boldsymbol{M}(U), N(U)), \\
U-\rightarrow \Lambda^{p} \boldsymbol{M}(U), \quad \text { respectively, }
\end{gathered}
$$

allow to define the tensor sheaf $\boldsymbol{M}_{\boldsymbol{\sigma}_{X}} \boldsymbol{N}$, the homomorphism sheaf $\operatorname{Hom}_{\boldsymbol{O}_{X}}(\boldsymbol{M}, \boldsymbol{N})$ and the $p$-th exterior power sheaf $\Lambda^{p} \boldsymbol{M}$. Note for this purpose that $\boldsymbol{M}(U) \emptyset_{X}(U) \boldsymbol{N}(U)$ is $\mathbf{L}(\boldsymbol{p})$-graded, its homogeneous component of degree $\vec{l}$ being spanned by all $m \infty n$ with $m \in \boldsymbol{M}(U)_{\vec{x}}, n \in \boldsymbol{N}(U)_{\vec{y}}$ and $\vec{x}+\vec{y}=\vec{l}$. The $\mathbf{L}(\boldsymbol{p})$-grading on $\Lambda^{p} \boldsymbol{M}(U)$ is given by a similar procedure. The homogeneous component of $\operatorname{HOM}(\boldsymbol{M}(U), \boldsymbol{N}(U))$ of degree $\vec{l}$ consists by definition of all morphisms of $\boldsymbol{O}_{\boldsymbol{X}}(U)$-modules of degree $\vec{l}$.

Clearly, $\boldsymbol{M}(\vec{l})=\boldsymbol{O}_{\boldsymbol{X}}(\vec{l}) \boldsymbol{\boldsymbol { O }}_{\mathbf{X}} \boldsymbol{M}$, moreover $\operatorname{coh}(\boldsymbol{X})$ is stable under the above operations.

1.8. Following Serre [31] (see also [17]) we define a sheafification functor $\sim: \operatorname{Mod}^{\mathbf{L}(\boldsymbol{p})}(S) \rightarrow \operatorname{Mod}^{\mathbf{L}(\boldsymbol{p})}\left(\boldsymbol{O}_{\boldsymbol{X}}\right), M-\rightarrow \tilde{M}$, where $\tilde{M}$ is the $\mathbf{L}(\boldsymbol{p})$-graded $\boldsymbol{O}_{\boldsymbol{X}}$-module attached to the presheaf

$$
\mathrm{D}(f) \rightarrow M_{f}, f \in S \text { homogeneous , }
$$

of $\mathbf{L}(\boldsymbol{p})$-graded $\boldsymbol{O}_{X}$-modules. Hence $M_{f}$ consists of all fractions $\frac{m}{f^{n}}(m \in M, n \in N)$. In contrast to the traditional approach, we do not restrict to the zero-component of $M_{f}$. As in (1.5.4) we get

$$
\Gamma(\mathrm{D}(f), \tilde{M})=M_{f}, \quad \text { if } f \in S_{+} \text {is homogeneous. }
$$

Again in contrast to [8], [10], [6], we have Serre's theorem (compare [31], [17]), which allows to remove all pathologies of weighted projective spaces encountered in these papers (compare for instance Section 3 of [6]):

Serre's theorem. Let $\boldsymbol{X}$ denote either $\mathbf{P}_{n}(p)$ or $\boldsymbol{C}(\boldsymbol{p}, \lambda)$, accordingly $S$ be either the $\mathbf{L}(\boldsymbol{p})$-graded algebra $S(\boldsymbol{p})$ or $S(\boldsymbol{p}, \boldsymbol{\lambda})$. Then

(i) Sheafification $\sim \bmod ^{\mathbf{L}_{+}}(S)-\rightarrow \operatorname{coh}(X), M-\rightarrow \tilde{M}$ is an exact functor, which admits

$$
\Gamma_{+}: \operatorname{coh}(\boldsymbol{X})-\rightarrow \bmod ^{\mathbf{L}_{+}}(S), \boldsymbol{M}-\rightarrow \underset{\vec{l} \geq 0}{\dagger} \Gamma(\boldsymbol{X}, \boldsymbol{M})_{\vec{l}}
$$

as a right adjoint. $\Gamma_{+}$is a full embedding satisfying $\Gamma_{+}(\boldsymbol{M})^{\sim}=\boldsymbol{M}$ for all $\boldsymbol{M} \in \operatorname{coh}(\boldsymbol{X})$.

(ii) Sheafification annihilates exactly the $M \in \bmod ^{\mathbf{L}_{+}}(S)$, which have finite length, and induces an equivalence 


$$
\bmod ^{\mathbf{L}_{+}}(S) / \bmod _{0}^{\mathbf{L}_{+}}(S)-\rightarrow \operatorname{coh}(X), \quad M-\rightarrow \tilde{M}
$$

of abelian categories.

(iii) The full subcategory $\boldsymbol{A}$ of $\bmod ^{\mathbf{L}_{+}}(S)$ consisting of all $M$, satisfying

$\operatorname{Hom}(E, M)=0=\operatorname{Ext}_{S}^{1}(E, M)$ for all simple objects $E$ in $\bmod ^{\mathbf{L}_{+}}(S)$,

is an abelian category. Moreover $\sim$ and $\Gamma_{+}$induce mutually inverse equivalences $: \boldsymbol{A} \rightarrow \rightarrow \operatorname{coh}(\boldsymbol{X})$ and $\Gamma_{+}: \operatorname{coh}(\boldsymbol{X})-\rightarrow \boldsymbol{A}$.

(iv) The passage $\operatorname{coh}(\boldsymbol{X})-\rightarrow \bmod ^{\mathbf{L}(p)}\left(\boldsymbol{O}_{X, \xi}\right), \boldsymbol{F} \rightarrow \boldsymbol{F}_{\xi}$ is an exact functor and induces an equivalence between $\operatorname{coh}(\boldsymbol{X}) / \operatorname{Ker} T$ and the category $\bmod (k(X))$ of finite dimensional vector spaces over the function field of $\boldsymbol{X}$. If $\boldsymbol{X}=C(p, \lambda), \operatorname{Ker} T$ is the subcategory $\mathrm{coh}_{0}(\boldsymbol{X})$ of all finite length coherent sheaves.

Proof: Since for a quasicoherent sheaf $\boldsymbol{F}$, each section $s \in \Gamma(\mathrm{D}(f), \boldsymbol{F})$ extends to a global section of $\boldsymbol{F}$ - up to multiplication with a suitable power of $f$ - each quasicoherent (coherent) sheaf on $\boldsymbol{X}$ has the form $\tilde{M}$ for some $M \in \operatorname{Mod}^{\mathbf{L}(p)}(S), M \in \bmod ^{\mathbf{L}(p)}(S)$, respectively.

For $f$ a homogeneous element of $S_{+}, \mathrm{D}(f)$ is an affine open subset of $\boldsymbol{X}$. By definition this means that the functor

$$
\operatorname{Mod}^{\mathbf{L}(p)}\left(\boldsymbol{O}_{\mathrm{D}(f)}\right)-\rightarrow \operatorname{Mod}^{\mathbf{L}(p)}\left(S_{f}\right), M-\rightarrow \Gamma(\mathrm{D}(f), \boldsymbol{M})
$$

induces category equivalences

$$
\Gamma: \mathrm{Q} c o h(\mathrm{D}(f))-\rightarrow \operatorname{Mod}^{\mathbf{L}(p)}\left(S_{f}\right) \text { and } \Gamma: \operatorname{coh}(\mathrm{D}(f))-\rightarrow \bmod ^{\mathbf{L}}(\boldsymbol{p})\left(S_{f}\right),
$$

respectively, with inverse given by sheafification $M-\rightarrow \tilde{M}$ similar to the one explained before. Since

$$
\Gamma(\mathrm{D}(f), \tilde{M})=M_{f},
$$

we obtain the formula

$$
\tilde{M}_{\mathrm{l}_{\mathrm{D}}}=\left(M_{f}\right)^{\tilde{}}
$$

as an equivalent assertion.

If $\boldsymbol{F}$ is quasi-coherent, thus $\boldsymbol{F}=\tilde{M}$, this proves by restriction to the various $\mathrm{D}(f)$ that

$$
\beta_{\boldsymbol{F}}:(\Gamma \boldsymbol{F})^{\tilde{-}} \rightarrow \boldsymbol{F},
$$

given by the maps

$$
\beta_{\mathrm{D}}:(\Gamma \boldsymbol{F})_{f}-\rightarrow \Gamma(\mathrm{D}, \boldsymbol{F}), \quad \frac{s}{f^{n}}-\rightarrow \frac{1}{f^{n}} s_{\mathrm{I}_{\mathrm{D}}}
$$


is an isomorphism of sheaves.

Moreover, given $M \in \operatorname{Mod}^{\mathbf{L}(p)}(S), \quad F \in \operatorname{Mod}^{\mathbf{L}(\boldsymbol{p})}\left(\boldsymbol{O}_{X}\right)$, the map

$$
\phi_{M}: \operatorname{Hom}(M, \Gamma \boldsymbol{F})-\rightarrow \operatorname{Hom}(\tilde{M}, \boldsymbol{F}), u-\rightarrow \beta_{\boldsymbol{F}^{\circ}} \tilde{u}
$$

is an isomorphism for each $M=S(\vec{l})$. Viewing both expressions as functors in $M$, this clearly implies that $\phi_{M}$ is always an isomorphism. This proves adjointness.

In order to prove assertion (iii), we first show that $\bmod _{0}^{\mathbf{L}_{+}}(S)$ is localizing in $\bmod ^{\mathbf{L}_{+}}(S)$, which means by definition that the natural functor

$$
T: \bmod ^{\mathbf{L}_{+}}(S)-\rightarrow \bmod ^{\mathbf{L}_{+}}(S) / \bmod _{0}^{\mathbf{L}_{+}}(S) \quad, \quad M-\rightarrow M
$$

has a left adjoint. According to ( [12], p. 372) this amounts to verify the following property $(*)$ for the full subcategory $\boldsymbol{A}$ of $\bmod ^{\mathbf{L}_{+}}(S)$, consisting of all modules $M$ with $\operatorname{Hom}_{S}(E, M)=0=\operatorname{Ext}_{S}^{1}(E, M)$ for all simple $E$ in $\bmod ^{\mathbf{L}_{+}}(S)$ :

(*) Each $M$ in $\bmod ^{\mathbf{L}_{+}}(S)$ has a submodule $M^{\prime}$, maximal among all submodules of finite length. Moreover if $M^{\prime}=0$, there is an exact sequence $0 \rightarrow M-\rightarrow A \rightarrow F-\rightarrow 0$ with $A \in A$ and $F \in \bmod _{0}^{\mathbf{L}_{+}}(S)$.

The first assertion follows since $S$ is noetherian. We note that - if $k=S / S_{+}$- the simple $S$-modules are - up to isomorphism - of the form $k(\vec{l})$, moreover $\operatorname{Ext}_{S}^{1}(k(\vec{l}), k(\vec{m})) \neq 0$ implies $\vec{l} \leq \vec{m}$. From the graded Koszul complex, attached to the regular sequence $X_{0}, X_{1}, \ldots, X_{n}$ for $S=S(\boldsymbol{p})$ and $x_{0}, x_{1}$ for $S=S(\boldsymbol{p}, \lambda)$, respectively, we deduce that the set of all $\vec{l} \in \mathbf{L}_{+}$with $\operatorname{Ext}^{1}(k(\vec{l}), M) \neq 0$, hence also its closure $E(M)$ with respect to predecessors, is finite. We are now going to prove our second assertion by induction on the cardinality $e(M)$ of $E(M)$. If $e(M)=0$, we are done. Otherwise, there is a non-split exact sequence $0 \rightarrow M-\rightarrow \bar{M}-\rightarrow k(\vec{m})-\rightarrow 0$ with $\vec{m}$ maximal in $E(M)$. We infer from the exactness of

$0-\rightarrow \operatorname{Hom}_{S}(k(\vec{l}), k(\vec{m}))-\rightarrow \operatorname{Ext}^{1}(k(\vec{l}), M)-\rightarrow \operatorname{Ext}^{1}(k(\vec{l}), \bar{M})-\rightarrow \operatorname{Ext}^{1}(k(\vec{l}), k(\vec{m}))$

that $E(\bar{M}) \subset E(M)$, moreover that $\operatorname{dim}_{k} \operatorname{Ext}^{1}(k(\vec{m}), \bar{M})<\operatorname{dim}_{k} \operatorname{Ext}^{1}(k(\vec{m}), M)$. Repeating this process, we may assume $e(\bar{M})<e(M)$. Since $\bar{M}$ has no simple submodules, the induction hypothesis applies to $\bar{M}$, so $\bar{M}$, hence $M$, embeds into some $A \in A$ with a finite length cokernel.

We are now going to prove that $\Gamma_{+}(\boldsymbol{F})$ is a finitely generated $\mathbf{L}_{+}$-graded $S$-module: By the previous argument, $\boldsymbol{F}=\tilde{M}$ for some $M \in A$. This yields an exact sequence $0-\rightarrow M-\rightarrow \Gamma_{+}(\tilde{M}) \rightarrow H \rightarrow 0$, where each finitely generated submodule of $H$ has finite length. If $H \neq 0$, we find a simple submodule $E$ of $H$. Since $M \in A, E$ embeds into $\Gamma_{+}(\tilde{M})$, which is impossible by (1.8.6), since $\tilde{S}=0$. Note for this purpose that $\tilde{M}=0$ if 
and only if $M_{f}=0$ for some homogeneous $f \in S_{+}$.

1.8.1. Corollary. All homomorphism spaces of $\operatorname{coh}(\boldsymbol{X})$ are finite dimensional over $k$; in particular $\operatorname{coh}(\boldsymbol{X})$ is a Krull-Schmidt category, i.e. each $\boldsymbol{F} \in \operatorname{coh}(\boldsymbol{X})$ has a decomposition $\boldsymbol{F}=\boldsymbol{F}_{1} \dashv \cdots \multimap \boldsymbol{F}_{n}$ where each $\boldsymbol{F}_{i}$ is indecomposable with local endomorphism ring. Moreover, we have $\operatorname{Hom}(\boldsymbol{O}(\vec{x}), \boldsymbol{O}(\vec{y}))=S_{\vec{y}-\vec{x}}$ for all $\vec{x}, \vec{y} \in \mathbf{L}(\boldsymbol{p})$.

1.8.2. Corollary. If $K$ denotes the function field of $\boldsymbol{X}$, the formula $\mathrm{r}(\boldsymbol{F})=\operatorname{dim}_{K}\left(\boldsymbol{F}_{\xi}\right)_{0}$ defines a linear form $\mathrm{r}: K_{0}(\boldsymbol{X})-\rightarrow \mathbf{Z}$, called the rank function. Moreover if $\boldsymbol{X}=\boldsymbol{C}(\boldsymbol{p}, \boldsymbol{\lambda})$, $\mathrm{r}(\boldsymbol{F})=0$ if and only if $\boldsymbol{F}$ has finite length.

1.8.3. Corollary. Each coherent sheaf $\boldsymbol{F} \in \operatorname{coh}(\boldsymbol{X})$ has an exact resolution

$$
\cdots-\rightarrow L_{2} \rightarrow L_{1} \rightarrow L_{0}-\rightarrow F-\rightarrow 0,
$$

where each $L_{i}$ is a finite direct sum of sheaves of the form $O_{X}(\vec{x})$. Moreover for $\boldsymbol{X}=\mathbf{P}_{n}(\boldsymbol{p})$ we may assume $\boldsymbol{L}_{j}=0$ for $j \geq n+2$.

Proof: Use gl.dim $S(\boldsymbol{p})=n+1$.

Actually as may be derived from our comparison theorem in Section 3, a stronger result holds true: We may assume $L_{j}=0$ for $j \geq n+1$ or $\boldsymbol{L}_{j}=0$ for $j \geq 2$ according as $\boldsymbol{X}=\mathbf{P}_{n}(\boldsymbol{p})$ or $\boldsymbol{X}=\boldsymbol{C}(\boldsymbol{p}, \boldsymbol{\lambda})$.

1.9. A coherent sheaf $\boldsymbol{F} \in \operatorname{coh}(\boldsymbol{X})$ is called a vector bundle if $\boldsymbol{F}$ is locally free, i. e. $\boldsymbol{X}$ can be covered by open subsets $U$ such that

$$
\left.\boldsymbol{F}\right|_{U}=\left.\bigoplus_{i=1}^{n} \boldsymbol{O}_{\boldsymbol{X}}\left(\vec{l}_{i}\right)\right|_{U}
$$

for suitably chosen $\vec{l}_{i}$, depending on $U$. The number $n$ is just the rank of $\boldsymbol{F}$, defined before.

We denote the full subcategory of $\operatorname{coh}(\boldsymbol{X})$, consisting of all vector bundles on $\boldsymbol{X}$, by $\operatorname{vect}(X)$. Usually we use letters as $F, G, \ldots$ to denote vector bundles. Since all stalks $\boldsymbol{O}_{\boldsymbol{X}, x}$ have graded global dimension $\mathrm{n}\left(1\right.$, resp.) according as $\boldsymbol{X}=\mathbf{P}_{n}(\boldsymbol{p})$ or $\boldsymbol{X}=\boldsymbol{C}(\boldsymbol{p}, \lambda)$, each coherent sheaf $\boldsymbol{F}$ has an (exact) resolution

$$
\begin{aligned}
& 0-\rightarrow L_{n} \rightarrow L_{n-1}-\rightarrow \cdots-\rightarrow L_{0} \rightarrow \boldsymbol{F}-\rightarrow 0 \quad\left(\boldsymbol{X}=\mathbf{P}_{n}(\boldsymbol{p})\right), \\
& 0-\rightarrow L_{1}-\rightarrow L_{0}-\rightarrow \boldsymbol{F}-\rightarrow 0 \quad(\boldsymbol{X}=C(\boldsymbol{p}, \lambda))
\end{aligned}
$$

by vector bundles $L_{i}$. 


\section{Serre duality and Riemann-Roch theorem}

Throughout this section, $\boldsymbol{C}$ denotes the curve $\boldsymbol{C}(\boldsymbol{p}, \boldsymbol{\lambda})$ in weighted projective space $\mathbf{P}_{n}(p)$.

We recall that the Picard group $\operatorname{Pic}(\boldsymbol{C})$ of $\boldsymbol{C}$ consists of all line bundles (= rank one vector bundles) on $\boldsymbol{C}$ with multiplication induced by the tensor product.

2.1. Proposition. The map $\vec{l}-\rightarrow \boldsymbol{O}_{C}(\vec{l})$ allows to identify the graduation group $\mathbf{L}(\boldsymbol{p})$ with the Picard group $\operatorname{Pic}(\boldsymbol{C})$.

Proof: $S=S(\boldsymbol{p}, \lambda)$ is $\mathbf{L}(\boldsymbol{p})$-graded factorial by Proposition 1.3.

In the following $\mathrm{D}$ stands for the formation of the k-dual.

2.2. Serre duality. For $\boldsymbol{F}, \boldsymbol{G} \in \operatorname{coh}(\boldsymbol{C})$ we have an isomorphism

$$
\operatorname{DExt}^{1}(\boldsymbol{F}, \boldsymbol{G})-\rightarrow \operatorname{Hom}(\boldsymbol{G}, \boldsymbol{F}(\vec{\omega})),
$$

which is functorial in $\boldsymbol{F}$ and $\boldsymbol{G}$, where $\vec{\omega}=(n-1) \vec{c}-\sum_{i=0}^{n} \vec{x}_{i}$ is the dualizing element of $\mathbf{L}(\boldsymbol{p})$.

Proof. Proceeding as in [18], we first calculate the $\mathbf{L}(p)$-graded Cech cohomology groups of $\boldsymbol{X}=\mathbf{P}_{n}(\boldsymbol{p})$ attached to the affine open covering, consisting of all $\mathrm{D}\left(X_{i}\right), \quad i=0, \ldots, n$. By means of the regular sequence $X_{0}, \ldots, X_{n}$ for $S(\boldsymbol{p})$ it then follows that

$$
\mathrm{DH}^{i}\left(\boldsymbol{X}, \boldsymbol{O}_{\boldsymbol{X}}\right)=\mathrm{H}^{n-i}\left(\boldsymbol{X}, \boldsymbol{O}_{\boldsymbol{X}}\left(-\sum_{i=0}^{n} \vec{x}_{i}\right)\right)
$$

for each $i=0, \ldots, n$. Note for this purpose that for each $M \in \operatorname{Mod}^{\mathrm{L}(p)}(S), \mathrm{D} M$ is the $\mathbf{L}(\boldsymbol{p})$-graded module with components $(\mathrm{D} M)_{\vec{x}}=\mathrm{D}\left(M_{-\vec{x}}\right) . \boldsymbol{O}\left(-\sum_{i=0}^{n} \vec{x}_{i}\right)$ occurs in (2.1.1) since $S\left(-\sum_{i=0}^{n} \vec{x}_{i}\right)$ is the last non-zero term of the $\mathbf{L}(\boldsymbol{p})$-graded Koszul complex attached to the regular sequence $X_{0}, \ldots, X_{n}$.

Using ( [15], théorème 5.9.2) and ( [23], proposition 7.17), it follows that Cech cohomology coincides with sheaf cohomology, calculated by means of injective resolutions either in $\operatorname{Mod}^{\mathbf{L}(p)}\left(\boldsymbol{O}_{X}\right)$ or in $\mathrm{Qcoh}(\boldsymbol{X})$.

Since the system of defining equations $f_{i}=X_{i}^{p_{i}}-X_{1}^{p_{1}}+\lambda_{i} X_{0}^{p_{0}},(i=2, \ldots, n)$ forms a regular sequence of homogeneous elements of $S(\boldsymbol{p})$, each of degree $\vec{c}$, it follows by standard arguments (see [6], p.57) the existence of an isomorphism of graded $S$ - 
modules $(S=S(\boldsymbol{p}, \lambda))$

$$
\mathrm{DH}^{1}\left(\boldsymbol{C}, \boldsymbol{O}_{C}\right)=\Gamma\left(\boldsymbol{C}, \boldsymbol{O}_{C}(\vec{\omega})\right),
$$

with $\vec{\omega}=(n-1) \vec{c}-\sum_{i=0}^{n} \vec{x}_{i}$. In particular for each $\vec{x} \in \mathbf{L}(\boldsymbol{p})$ we obtain a uniquely defined $\eta_{\vec{x}} \in \operatorname{DExt}^{1}\left(\boldsymbol{O}_{C}(\vec{x}), \boldsymbol{O}_{C}(\vec{x}+\vec{\omega})\right)$, corresponding up to twist to the identity in $\operatorname{Hom}\left(\boldsymbol{O}_{\boldsymbol{C}}, \boldsymbol{O}_{\boldsymbol{C}}\right)$ by means of (2.2.2). Yoneda's lemma yields natural transformations

$$
\eta_{\vec{x}}: \operatorname{Hom}\left(\boldsymbol{O}_{C}(\vec{x}),-\right)-\rightarrow \operatorname{DExt}^{1}\left(-, \boldsymbol{O}_{C}(\vec{x}+\vec{\omega})\right),
$$

functorial with respect to morphisms $\boldsymbol{O}_{C}(\vec{x})-\rightarrow \boldsymbol{O}_{C}(\vec{y})$. Actually, we may use the family of all $\eta_{\vec{x}}$ to define natural morphisms

$$
\eta_{\boldsymbol{F}, \boldsymbol{G}}: \operatorname{Hom}(\boldsymbol{F}, \boldsymbol{G})-\rightarrow \operatorname{DExt}^{1}(\boldsymbol{G}, \boldsymbol{F}(\vec{\omega}))
$$

for each pair $\boldsymbol{F}, \boldsymbol{G}$ of coherent sheaves on $\boldsymbol{C}$, working with resolutions of $\boldsymbol{F}$ by direct sums of twisted structure sheaves, induced by free resolutions of $\Gamma_{+}(\boldsymbol{F})$ in $\operatorname{Mod}^{L_{+}}(S)$.

Since all stalks of $\boldsymbol{C}$ have graded global dimension one, each quasi-coherent sheaf $\boldsymbol{M}$ has injective dimension at most one (cf. [23], proposition 7.17), so Ext $^{2}$ vanishes. By standard arguments (see [24], p. 240) one first proves that $\eta_{\boldsymbol{F}, \boldsymbol{G}}$ is an isomorphism if $\boldsymbol{F}=\boldsymbol{O}_{C}(\vec{x})$ and $\boldsymbol{G}$ is arbitrary; combining right exactness of Ext ${ }^{1}$ with Corollary 1.8.3 now proves the assertion.

2.3. Corollary. The category $\operatorname{coh}(\boldsymbol{C})$ has almost-split sequences. Moreover, twisting $\boldsymbol{F} \rightarrow \boldsymbol{F}(\vec{\omega})$ with the dualizing element serves as the Auslander-Reiten translation for $\operatorname{coh}(\boldsymbol{C})$.

Proof: The assertion means that for each indecomposable coherent sheaf $\boldsymbol{F}$ there exists a non-split exact sequence

$$
\eta: 0-\rightarrow \boldsymbol{F}(\vec{\omega}) \stackrel{u}{\rightarrow} \boldsymbol{G}-\stackrel{v}{\rightarrow} \boldsymbol{F}-\rightarrow 0
$$

such that for each indecomposable sheaf $\boldsymbol{X}$, each non-isomorphism $f: X-\rightarrow \boldsymbol{F}$ lifts to $\boldsymbol{G}$ via $v$.

Since $\boldsymbol{F}(\vec{\omega})$ has a local endomorphism ring, $\operatorname{Hom}(-, \boldsymbol{F}(\vec{\omega}))$ - as an abelian group valued additive functor on $\operatorname{coh}(\boldsymbol{C})^{o p}$ - has a (unique) simple quotient $\boldsymbol{H}$, necessarily with $\boldsymbol{H}(\boldsymbol{F}(\vec{\omega})) \neq 0$. By Serre duality $\mathrm{DH}$ becomes a simple subfunctor of $\operatorname{Ext}^{1}(\boldsymbol{F},-)$ with $\operatorname{DH}(\boldsymbol{F}(\vec{\omega})) \neq 0$. Each non-zero $\eta \in \mathrm{D} \boldsymbol{H}(\boldsymbol{F}(\vec{\omega})) \subset \operatorname{Ext}^{1}(\boldsymbol{F}, \boldsymbol{F}(\vec{\omega}))$ represents an almostsplit sequence. 
For further information on almost-split sequences we refer to [2], [14].

For non-singular projective curves the above proof is due to Schofield. Independently, alternative existence proofs were given by Auslander and Reiten, based on the methods of [3], also by the authors using their comparison theorem (see Section 3). If the weight sequence determines a Dynkin diagram, i.e. for $\sum_{i=0}^{n} \frac{1}{p_{i}}>n-1$, Propositions 2.2 and 2.3 are covered by [26].

As we may deduce from Serre's theorem, each coherent sheaf $\boldsymbol{F}$ has a greatest subsheaf of finite length $t \boldsymbol{F}$, called the torsion sheaf of $\boldsymbol{F} . \boldsymbol{F} / t \boldsymbol{F}$ has no simple subsheaves, so is torsion-free. Note that $\boldsymbol{F}=t \boldsymbol{F}$ if and only if $\boldsymbol{F}$ has finite support $\left\{x \in \boldsymbol{C} \mid \boldsymbol{F}_{x} \neq 0\right\}$.

2.4. Proposition. Each coherent sheaf $\boldsymbol{F}$ on $\boldsymbol{C}$ has a decomposition $\boldsymbol{F}=t \boldsymbol{F}$ to $\boldsymbol{F}$, where t $\boldsymbol{F}$ is the torsion sheaf of $\boldsymbol{F}$ and $F \tilde{=} \boldsymbol{F} / t \boldsymbol{F}$ is a vector bundle. In particular, each subsheaf of a vector bundle is a vector bundle again.

Proof: We claim that the sequence

$$
0-\rightarrow t \boldsymbol{F}-\rightarrow \boldsymbol{F}-\rightarrow \boldsymbol{F} / t \boldsymbol{F}-\rightarrow 0
$$

splits, moreover that $\boldsymbol{F} / t \boldsymbol{F}$ is locally free. Since $t \boldsymbol{F}$ has finite support, both properties are of a local character. Passing to $\bmod ^{\mathbf{L}(p)}\left(S_{f}\right)$ the proof of the corresponding assertions follows from the fact that $S_{f}$ is an $\mathbf{L}(\boldsymbol{p})$-graded Dedekind domain. Note for this purpose that all stalks of $\boldsymbol{O}_{\boldsymbol{C}}$ are graded discrete valuation domains (cf. (1.6)).

For $x \in C$ let $\operatorname{coh}^{\mathbf{L}(p)}(\boldsymbol{C})_{x}$ denote the full subcategory of coherent sheaves with support in $x$. Clearly,

$$
\operatorname{coh}(\boldsymbol{C})_{x}-\rightarrow \bmod _{0}\left(\boldsymbol{O}_{\boldsymbol{C}, x}\right), \quad \boldsymbol{F} \rightarrow \boldsymbol{F}_{x}
$$

defines an equivalence; moreover $\operatorname{coh}_{o}(\boldsymbol{C})$, the category of all torsion sheaves decomposes into $\underset{x \in C}{\Perp} \operatorname{coh}(\boldsymbol{C})_{x}$.

2.5. Proposition. The category $\operatorname{coh}_{o}(\boldsymbol{C})$ of torsion sheaves on $\boldsymbol{C}$ decomposes into a coproduct $\underset{x \in C}{\Perp} \bmod _{0}{ }^{\mathbf{L}(p)}\left(\boldsymbol{O}_{C},{ }_{x}\right)$ of uniserial categories. The number of isomorphism classes of simple modules in $\bmod _{0}\left(\boldsymbol{O}_{\boldsymbol{C}, x}\right)$ is given by the weight $\boldsymbol{p}(x)$ of $x$.

Proof. Each stalk $\boldsymbol{O}_{C, x}$ is a graded discrete valuation ring, its number of simple graded modules is given by the weight $p(x)$ of $x$. 
For later use we give the following explicit description of simple sheaves on $C$ :

If $x=\left[x_{0}, x_{1}, \ldots, x_{n}\right]$ is an ordinary point and $\lambda=\frac{x_{1}^{p_{1}}}{x_{0}^{p_{0}}}$, multiplication with $u=X_{1}^{p_{1}}-\lambda X_{0}^{p_{0}}$ leads to an exact sequence

$$
0-\rightarrow \boldsymbol{O}_{C}-\stackrel{u}{\rightarrow} \boldsymbol{O}_{C}(\vec{c})-\rightarrow S-\rightarrow 0
$$

where $S$ is the unique simple sheaf concentrated at $x$.

By contrast, if $x$ is exceptional with $x_{i}=0$, multiplication by $X_{i}$ leads to exact sequences

$$
0-\rightarrow \boldsymbol{O}_{\boldsymbol{C}}\left(j \vec{x}_{i}\right) \stackrel{X_{i}}{\rightarrow} \boldsymbol{O}_{\boldsymbol{C}}\left((j+1) \vec{x}_{i}\right)-\rightarrow \boldsymbol{S}_{i, j}-\rightarrow 0, \quad j \in \mathbf{Z} / p_{i} \mathbf{Z},
$$

defining $p_{i}$ mutually non-isomorphic simple sheaves concentrated at $x$.

As is easily checked,

$$
\boldsymbol{S}(\vec{x}) \tilde{=} S \text { for all } \vec{x} \in \mathbf{L}(\boldsymbol{p}),
$$

if $\boldsymbol{S}$ is ordinary simple. For the exceptional simple sheaves

$$
\boldsymbol{S}_{i, j}(\vec{x}) \tilde{=} \boldsymbol{S}_{i, j+l_{i}}, \quad \text { for } \vec{x}=\sum_{r=0}^{n} l_{\mathrm{r}} \vec{x}_{\mathrm{r}} .
$$

In particular, $S_{i, j}(\vec{\omega})=S_{i, j-1}$.

So the classification of (indecomposable) coherent sheaves reduces to the classification of vector bundles. Here, the existence of a line bundle filtration serves as the basic tool:

2.6. Proposition. Each vector bundle $F$ on $C$ has a filtration

$$
0=F_{0} \subset F_{1} \subset \cdots \subset F_{n}=F,
$$

whose factors $F_{i} / F_{i-1}$ are line bundles, hence of the form $\boldsymbol{O}_{\boldsymbol{C}}\left(\vec{l}_{i}\right)$ for suitably chosen $\vec{l}_{i} \in \mathbf{L}(\boldsymbol{p})$.

Proof. We proceed by induction on $\mathrm{r}(F)$. As follows from Serre's theorem, we may assume by a suitable twist that $\operatorname{Hom}\left(\boldsymbol{O}_{\boldsymbol{C}}, \boldsymbol{F}\right) \neq 0$, hence $\boldsymbol{O}_{C}$ may be viewed as a subsheaf of $\boldsymbol{F}$. If $F_{1} \subset F$ is chosen with $F_{1} / \boldsymbol{O}_{\boldsymbol{C}}=t\left(\boldsymbol{F} / \boldsymbol{O}_{\boldsymbol{C}}\right), F_{1}$ is clearly a line bundle and even a subbundle of $F$, i. e. $F / F_{1}$ is a vector bundle again. The assertion now follows from the induction hypothesis. 
2.7. Corollary. Let $x \in C$. If $F$ is a vector bundle on $C$ of rank $n$, there is an exact sequence

$$
0 \rightarrow F \rightarrow \biguplus_{i=1}^{n} L_{i} \rightarrow H \rightarrow 0
$$

in $\operatorname{coh}(\boldsymbol{C})$, where each $L_{i}$ is a line bundle and $\boldsymbol{H}$ is concentrated at $x$.

Proof. We proceed by induction on $n$. Choose an exact bundle sequence $0 \rightarrow F_{n-1} \rightarrow F \rightarrow L_{n} \rightarrow 0$ as in (2.6), where $L_{n}$ is a line bundle. By assumption $F_{n-1}$ embeds into a direct sum $\bigoplus_{i=1}^{n-1} L_{i}$ with cokernel concentrated at $x$. Replacing - if necessary - each $L_{i}$ by some $L_{i}(l \vec{c})$, by means of Serre duality we may assume that $\operatorname{Ext}^{1}\left(L_{n}, L_{j}\right)=0$ for all $j=1, \ldots, n$. Passage to the push-out

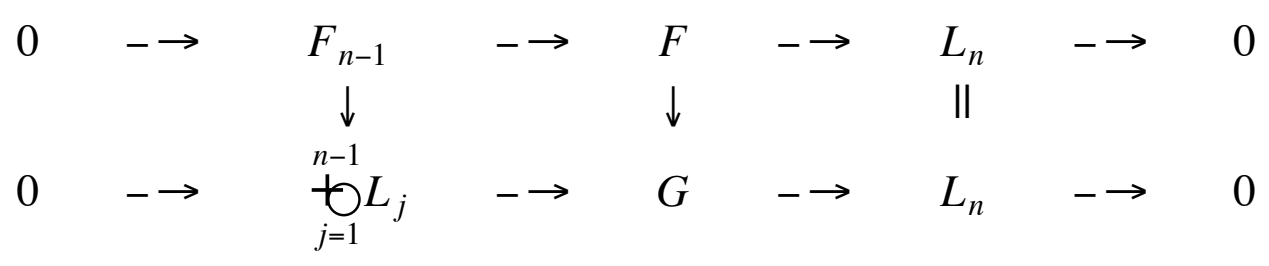

proves that $F$ embeds into $G \tilde{=} \bigoplus_{i=1}^{n} F_{i}$, again with cokernel concentrated at $x$.

As follows from the foregoing, the classes $\left[\boldsymbol{O}_{\boldsymbol{C}}\left(\vec{l}_{i}\right)\right]$ form a system of generators for the Grothendieck group $\mathrm{K}_{0}(C)$, which is defined as the Grothendieck group of $\operatorname{coh}(\boldsymbol{C})$ with respect to short exact sequences. The previous discussion shows that equivalently $\left[\boldsymbol{O}_{\boldsymbol{C}}\right]$ and the classes $[\boldsymbol{S}]$ of simple sheaves generate $\mathrm{K}_{0}(\boldsymbol{C})$. Actually the classes $\left[\boldsymbol{O}_{C}(\vec{x})\right]$, for $0 \leq \vec{x} \leq \vec{c}$, form a $\mathbf{Z}$-basis for $\mathrm{K}_{0}(C)$, as we will see later. As usual, we view linear forms on $\mathrm{K}_{0}(\boldsymbol{C})$ as functions on $\operatorname{coh}(\boldsymbol{C})$, which are additive on short exact sequences.

Since $\operatorname{Ext}^{2}$ vanishes on $\operatorname{coh}(\boldsymbol{C})$, the Euler characteristic given by

$$
\chi: \mathrm{K}_{0}(\boldsymbol{C})-\rightarrow \mathbf{Z} \quad, \quad[\boldsymbol{F}] \rightarrow \sum_{j=0}^{1}(-1)^{j} \operatorname{dim}_{k} \operatorname{Ext}^{j}\left(\boldsymbol{O}_{\boldsymbol{C}}, \boldsymbol{F}\right)
$$

is a linear form on $\mathrm{K}_{0}(\boldsymbol{C})$. We recall that $\delta: \mathbf{L}(\boldsymbol{p})-\rightarrow \mathbf{Z}$ is the group homomorphism, defined on generators by $\delta\left(\vec{x}_{i}\right)=\frac{p}{p_{i}}$, where $p=l \cdot c \cdot m \cdot\left(p_{0}, \ldots, p_{n}\right)$.

2.8. Proposition. There is a linear form $\mathrm{d}: \mathrm{K}_{0}(\boldsymbol{C})-\rightarrow \mathbf{Z}$, called the degree, which is uniquely determined by each of the following properties

(i) $\mathrm{d}\left(\boldsymbol{O}_{C}(\vec{x})\right)=\delta(\vec{x})$ for each $\vec{x} \in \mathbf{L}(\boldsymbol{p})$. 
(ii) $\mathrm{d}\left(\boldsymbol{O}_{C}\right)=0$, and $\mathrm{d}(\boldsymbol{S})=\frac{p}{\boldsymbol{p}(x)}$ if $\boldsymbol{S}$ is a simple sheaf, concentrated at $x$.

Proof. Let $\bar{\chi}(\boldsymbol{F})=\sum_{j=0}^{p-1} \chi(\boldsymbol{F}(-j \vec{\omega}))$. Using Serre duality, formulas (2.5.1) and (2.5.2) yield $\bar{\chi}(S)=p$ if $\boldsymbol{p}(x)=1$ and $\bar{\chi}\left(\boldsymbol{S}_{i, j}\right)=\frac{p}{p_{i}}$ for $0 \leq i \leq n, 1 \leq j \leq p_{i}$. Hence $\mathrm{d}: \mathrm{K}_{0}(\boldsymbol{C})-\rightarrow \mathbf{Z}$ given by

$$
\mathrm{d}(\boldsymbol{F})=\bar{\chi}(\boldsymbol{F})-\mathrm{r}(\boldsymbol{F}) \bar{\chi}\left(\boldsymbol{O}_{C}\right)
$$

satisfies condition (ii). Condition ( $i$ ) now follows by repeated use of exact sequences of type

$$
0-\rightarrow O_{C}(\vec{x})-\rightarrow O_{C}\left(\vec{x}+\vec{x}_{i}\right)-\rightarrow S_{i, 0}(\vec{x})-\rightarrow 0 .
$$

Actually with formula (2.8.2) we have proved the first assertion of Riemann-Roch's theorem:

2.9. Riemann-Roch theorem. The averaged Euler characteristic for $\boldsymbol{C}$ given by

$$
\bar{\chi}(\boldsymbol{F})=\sum_{j=0}^{p-1} \chi(\boldsymbol{F}(-j \vec{\omega}))
$$

satisfies

$$
\bar{\chi}(\boldsymbol{F})=\mathrm{r}(\boldsymbol{F}) \bar{\chi}\left(\boldsymbol{O}_{\boldsymbol{C}}\right)+\mathrm{d}(\boldsymbol{F}),
$$

in particular

$$
\bar{\chi}\left(\boldsymbol{O}_{C}(\vec{x})\right)=\bar{\chi}\left(\boldsymbol{O}_{C}\right)+\delta(\vec{x})
$$

holds true for each $\vec{x} \in \mathbf{L}(\boldsymbol{p})$. Moreover

$$
\frac{1}{p} \bar{\chi}\left(O_{C}\right)=-\frac{1}{2} \delta(\vec{\omega})=\frac{p}{2}\left(\sum_{i=0}^{n} \frac{1}{p_{i}}-(n-1)\right) .
$$

Proof: It remains to prove the last assertion. From Serre duality we get

$$
\chi\left(\boldsymbol{O}_{\boldsymbol{C}}(j \vec{\omega})\right)=\operatorname{dim}_{k} \operatorname{Hom}\left(\boldsymbol{O}_{\boldsymbol{C}}, \boldsymbol{O}_{\boldsymbol{C}}(j \vec{\omega})\right)-\operatorname{dim}_{k} \operatorname{Hom}\left(\boldsymbol{O}_{\boldsymbol{C}}(j \vec{\omega}), \boldsymbol{O}_{\boldsymbol{C}}(\vec{\omega})\right)
$$

for each integer $\mathrm{j}$. Consequently

$$
\bar{\chi}\left(\boldsymbol{O}_{C}\right)+\bar{\chi}\left(\boldsymbol{O}_{C}(p \vec{\omega})\right)=\sum_{j=-(p-1)}^{p} \chi\left(\boldsymbol{O}_{C}(j \vec{\omega})\right)=0,
$$

hence $2 \bar{\chi}\left(\boldsymbol{O}_{\boldsymbol{C}}\right)+p \delta(\vec{\omega})=0$. 
We have already seen that the function field $k(\boldsymbol{C})$ of $\boldsymbol{C}$ is the field $k(t)$ of rational functions in the indeterminate $t$. Hence it is in accordance with usual terminology to view $\boldsymbol{C}$ as a curve of genus zero. However, Riemann-Roch's theorem suggests to consider also the virtual genus of $\boldsymbol{C}$, given by

$$
g_{v}(\boldsymbol{C})=1-\frac{1}{p} \bar{\chi}\left(\boldsymbol{O}_{\boldsymbol{C}}\right)
$$

Accordingly,

$$
g_{v}(C)=1+\frac{1}{2} \delta(\vec{\omega})
$$

\section{Tilting from sheaves to modules}

In this section we investigate the interrelations between coherent sheaves over $\boldsymbol{C}=\boldsymbol{C}(\boldsymbol{p}, \lambda)$ and representations of a finite dimensional $k$-algebra $\Lambda$, arising as endomorphism algebra of a tilting sheaf on $\boldsymbol{C}$. In the spirit of Beilinson's Theorem [5] we show that the derived categories of $\operatorname{coh}(C)$ and $\bmod \left(\Lambda^{o p}\right)$, the category of finite dimensional right $\Lambda$-modules, are equivalent. The equivalence $\mathrm{D}^{b}(\operatorname{coh}(\boldsymbol{C})) \tilde{=} \mathrm{D}^{b}\left(\bmod \left(\Lambda^{o p}\right)\right)$ is made precise by a comparison result (Theorem 3.3) which states together with its consequences that the classification problems for $\operatorname{coh}(C)$ and $\bmod \left(\Lambda^{o p}\right)$ are basically equivalent.

For an abelian category $\boldsymbol{A}, \mathrm{D}^{b}(A)$ denotes the derived category of bounded complexes in $\boldsymbol{A}$. We refer to [34] and [23] for the definition and properties of triangulated and derived categories. We will consider $\boldsymbol{A}$ as a full subcategory of $\mathrm{D}^{b}(\boldsymbol{A})$ viewing $A \in \boldsymbol{A}$ as a complex concentrated at 0 . We only note that $\mathrm{D}^{b}(\boldsymbol{A})$ is equipped with a translation functor $T$ given by $\left(T\left(X^{\bullet}\right)\right)^{n}=X^{n+1}$ and $\left(T \mathrm{~d}_{X^{\bullet}}\right)^{n}=-\mathrm{d}_{X^{\bullet}}^{n+1}$.

3.1. Definition. A coherent sheaf $\boldsymbol{T}$ on $\boldsymbol{C}$ is called a tilting sheaf if the following properties hold:

(1) $\operatorname{Ext}^{1}(\boldsymbol{T}, \boldsymbol{T})=0$

(2) $\boldsymbol{T}$ generates $\mathrm{D}^{b}(\operatorname{coh}(\boldsymbol{C}))$ as a triangulated category, i.e. $\mathrm{D}^{b}(\operatorname{coh}(\boldsymbol{C}))$ is the smallest triangulated subcategory of $\mathrm{D}^{b}(\operatorname{coh}(\boldsymbol{C}))$ containing $\boldsymbol{T}$.

(3) $\operatorname{gl} . \operatorname{dim}(\operatorname{End}(\boldsymbol{T}))<\infty$.

Actually condition (3) is a consequence of (1) and (2), as we will prove elsewhere.

Let $\boldsymbol{T}$ be a tilting sheaf and let $\Lambda=\operatorname{End}(\boldsymbol{T}) . \boldsymbol{T}$ induces a functor

$$
F=\operatorname{Hom}(\boldsymbol{T},-): \mathrm{Q} \operatorname{coh}(\boldsymbol{C})-\rightarrow \operatorname{Mod}\left(\Lambda^{o p}\right), \quad \boldsymbol{F}-\rightarrow \operatorname{Hom}(\boldsymbol{T}, \boldsymbol{F}) .
$$

Since $\Lambda=\operatorname{End}(\boldsymbol{T})$, there is a functor 


$$
G=-\rtimes_{\lambda} \boldsymbol{T}: \operatorname{Mod}\left(\Lambda^{o p}\right)-\rightarrow \mathrm{Q} \operatorname{coh}(C) .
$$

with $G(\Lambda)=\boldsymbol{T}$, which is right exact and commutes with arbitrary direct sums. $G$ is uniquely determined up to isomorphism.

In the language of sheaves this functor is given as follows: For each open subset $U \subset C, T(U)$ is a $\Lambda-O_{C}(U)$-bimodule, and $M \rtimes_{\triangle} T$ is isomorphic to the sheaf associated with the presheaf $U \rightarrow M \rtimes_{\lambda} \boldsymbol{T}(U)_{O_{C}(U)}$, for each $M \in \operatorname{Mod}\left(\Lambda^{o p}\right)$.

Since $\mathrm{Q} \operatorname{coh}(\boldsymbol{C})$ has enough injectives and finite global dimension, the right derived functor of $\boldsymbol{F}$,

$$
R^{*} F: \mathrm{D}^{b}(\mathrm{Q} \operatorname{coh}(\boldsymbol{C}))-\rightarrow \mathrm{D}^{b}\left(\operatorname{Mod}\left(\Lambda^{o p}\right)\right)
$$

exists; since $\operatorname{Mod}\left(\Lambda^{o p}\right)$ has enough projectives and finite global dimension, the left derived functor of $\boldsymbol{G}$,

$$
L_{*} G: \mathrm{D}^{b}\left(\operatorname{Mod}\left(\Lambda^{o p}\right)\right)-\rightarrow \mathrm{D}^{b}(\mathrm{Q} \operatorname{coh}(\boldsymbol{C}))
$$

exists (see [34], [23] for the definition and properties of derived functors).

3.2. Theorem. Let $\boldsymbol{T} \in \operatorname{coh}(\boldsymbol{C})$ be a tilting sheaf. The functors

$$
R^{*} \operatorname{Hom}(\boldsymbol{T},-): \mathrm{D}^{b}(\operatorname{coh}(\boldsymbol{C}))-\rightarrow \mathrm{D}^{b}\left(\bmod \left(\Lambda^{o p}\right)\right)
$$

and

$$
L_{*}\left(-\rtimes_{\lambda} T\right): \mathrm{D}^{b}\left(\bmod \left(\Lambda^{o p}\right)\right)-\rightarrow \mathrm{D}^{b}(\operatorname{coh}(\boldsymbol{C}))
$$

define equivalences of triangulated categories, mutually inverse to each other.

Proof: $\mathrm{D}^{b}(\operatorname{coh}(\boldsymbol{C}))$ and $\mathrm{D}^{b}\left(\bmod \left(\Lambda^{o p}\right)\right)$ are full triangulated subcategories of $\mathrm{D}^{b}(\mathrm{Q} \operatorname{coh}(\boldsymbol{C}))$ and $\mathrm{D}^{b}\left(\operatorname{Mod}\left(\Lambda^{o p}\right)\right)$, respectively. Note that $\Lambda$ generates $\mathrm{D}^{b}\left(\bmod \left(\Lambda^{o p}\right)\right)$ as triangulated subcategory since $\Lambda$ has finite global dimension. $R^{*} F$ and $L_{*} G$ induce equivalences between the full subcategories $\{T\}$ and $\{\Lambda\}$ of $\operatorname{Qcoh}(\boldsymbol{C})$ and $\operatorname{Mod}\left(\Lambda^{o p}\right)$, respectively. Thus the assertion follows from Beilinson's Lemma [5].

The following theorem explains the equivalence $\mathrm{D}^{b}(\operatorname{coh}(\boldsymbol{C})) \tilde{=} \mathrm{D}^{b}\left(\bmod \left(\Lambda^{o p}\right)\right)$ in the spirit of tilting theory [20], [21], [7].

3.3. Theorem. Let $\boldsymbol{T}$ be a tilting sheaf. We denote by $X_{i} \subset \operatorname{coh}(\boldsymbol{C})(i \geq 0)$ the full subcategory of all $\boldsymbol{F}$ with $\operatorname{Ext}^{j}(\boldsymbol{T}, \boldsymbol{F})=0$ for all $j \neq i$ and by $Y_{i} \subset \bmod \left(\Lambda^{o p}\right)(i \geq 0)$ the full subcategory of all $M$ with $\operatorname{Tor}_{j}^{\wedge}(M, T)=0$ for all $j \neq i$.

The functors 


$$
\operatorname{Ext}^{i}(\boldsymbol{T},-): \boldsymbol{X}_{i}-\rightarrow \boldsymbol{Y}_{i}
$$

and

$$
\operatorname{Tor}_{i}^{\Lambda}(-, T): Y_{i}-\rightarrow X_{i}
$$

define equivalences, mutually inverse to each other.

Proof: Let $F=\operatorname{Hom}(\boldsymbol{T},-)$ and $G=-\rtimes_{\boldsymbol{T}} \boldsymbol{T} . R^{i} F$ and $L_{i} G$ denote the $i$-th right derived functor of $F$ and the $i$-th left derived functor of $G$, respectively.

For $X_{i} \in X_{i}$ we have $H^{j} R^{*} F\left(X_{i}\right) \tilde{=} R^{j} F\left(X_{i}\right)=0$ for $j \neq i$. Thus $R^{*} F\left(X_{i}\right) \tilde{=} T^{-i} R^{i}\left(X_{i}\right)$ in $\mathrm{D}^{b}\left(\bmod \left(\Lambda^{o p}\right)\right)$, where $T$ denotes the translation functor. We have $L_{j} G R^{i} F\left(X_{i}\right)=H^{j} T^{i} L_{*} G R^{*} F\left(X_{i}\right)$, which is isomorphic to $X_{i}$ for $j=i$ and 0 otherwise, hence $R^{i} F\left(X_{i}\right) \in \boldsymbol{Y}_{i}$. Similarly we obtain $L_{i} G\left(Y_{i}\right) \in \boldsymbol{X}_{i}$, and $R^{i} F L_{i} G\left(Y_{i}\right) \tilde{=} Y_{i}$ for $Y_{i} \in \boldsymbol{Y}_{i}$.

We remark, that $\boldsymbol{X}_{i}=0$, hence $\boldsymbol{Y}_{i}=0$ for all $i>2$ since $\operatorname{Ext}^{2}(-,-)=0$ in $\operatorname{coh}(\boldsymbol{C})$.

We now investigate, how far the subcategories $\left(X_{0}, X_{1}\right)$ and $\left(Y_{0}, Y_{1}\right)$ determine the categories $\operatorname{coh}(\boldsymbol{C})$ and $\bmod \left(\Lambda^{o p}\right)$, respectively.

3.4. Corollary. $\left(\boldsymbol{X}_{0}, \boldsymbol{X}_{1}\right)$ is a torsion theory for $\operatorname{coh}(\boldsymbol{C})$. In particular $\boldsymbol{X}_{0}$ is the full subcategory of all coherent sheaves generated by $\boldsymbol{T}$, i.e. having the form $\boldsymbol{T}^{n} / \boldsymbol{U}$.

Proof: Obviously, $\boldsymbol{X}_{0} \cap \boldsymbol{X}_{1}=0, \boldsymbol{X}_{1}$ is closed under subobjects, and $\boldsymbol{X}_{0}$ is closed under homomorphic images. It remains to show that every coherent sheaf $\boldsymbol{F}$ admits an exact sequence

$$
0-\rightarrow F_{0}-\rightarrow F-\rightarrow F_{1}-\rightarrow 0
$$

with $\boldsymbol{F}_{0} \in \boldsymbol{X}_{0}, \boldsymbol{F}_{1} \in \boldsymbol{X}_{1}$.

Since $\boldsymbol{F}$ is noetherian, there exist a greatest subsheaf $\boldsymbol{F}_{0}$ generated by $\boldsymbol{T}$. Thus $\boldsymbol{F}_{0} \in \boldsymbol{X}_{0}$ and $\boldsymbol{F}_{1}=\boldsymbol{F} / \boldsymbol{F}_{0} \in \boldsymbol{X}_{1}$ follows. Moreover this shows that $\boldsymbol{F} \in \boldsymbol{X}_{0}$ if and only if there exist an epimorphism $\boldsymbol{T}^{n} \rightarrow \boldsymbol{F}$.

If $\boldsymbol{F} \in \operatorname{coh}(\boldsymbol{C})$, and $0-\rightarrow \boldsymbol{F}_{0}-\rightarrow \boldsymbol{F} \rightarrow \rightarrow \boldsymbol{F}_{1} \rightarrow 0$ is exact with $\boldsymbol{F}_{0} \in \boldsymbol{X}_{0}$ and $\boldsymbol{F}_{1} \in \boldsymbol{X}_{1}$, $\operatorname{Hom}(\boldsymbol{T}, \boldsymbol{F}) \tilde{=} \operatorname{Hom}\left(\boldsymbol{T}, \boldsymbol{F}_{0}\right)$ and $\operatorname{Ext}^{1}(\boldsymbol{T}, \boldsymbol{F}) \tilde{=} \operatorname{Ext}^{1}\left(\boldsymbol{T}, \boldsymbol{F}_{1}\right)$. This yields:

3.5. Corollary. For each coherent sheaf $\boldsymbol{F}$ there is an exact sequence

$$
0-\rightarrow \operatorname{Hom}(\boldsymbol{T}, \boldsymbol{F}) \bowtie \boldsymbol{T}-\rightarrow \boldsymbol{F}-\rightarrow \operatorname{Tor}_{1}^{\Lambda}\left(\operatorname{Ext}^{1}(\boldsymbol{T}, \boldsymbol{F}), \boldsymbol{T}\right)-\rightarrow 0 .
$$


3.6. Corollary. $\operatorname{Tor}_{j}^{\wedge}\left(\operatorname{Ext}^{i}(\boldsymbol{T}, \boldsymbol{F}), \boldsymbol{T}\right)=0$ for all $i, j=0,1, i \neq j$ and all coherent sheaves $\boldsymbol{F}$.

Similar results hold true for $\bmod \left(\Lambda^{o p}\right)$ :

3.7. Proposition. For each module $M \in \bmod \left(\Lambda^{o p}\right)$, there exists an exact sequence

$$
0-\rightarrow \operatorname{Ext}^{1}\left(\boldsymbol{T}, \operatorname{Tor}_{1}^{\wedge}(M, T)\right) \rightarrow M-\rightarrow \operatorname{Hom}(\boldsymbol{T}, M \rtimes \boldsymbol{T})-\rightarrow 0 .
$$

Proof: Let $P_{2} \rightarrow P_{1} \rightarrow P_{0} \rightarrow M-\rightarrow 0$ be a projective resolution of $M$. Tensoring with $T$ leads to the complex

$$
P_{2} \rtimes_{\lambda} \boldsymbol{T}-\stackrel{u_{2}}{\rightarrow} P_{1} \rtimes_{0} \boldsymbol{T}-\stackrel{u_{1}}{\rightarrow} P_{0} \rtimes_{\lambda} \boldsymbol{T}-\stackrel{u_{0}}{\rightarrow} M \rtimes_{\lambda} \boldsymbol{T}-\rightarrow 0,
$$

which is not necessarily exact in $P_{1} \boldsymbol{T}$. Let $\boldsymbol{K}_{i}=\operatorname{ker} u_{i}$ and $\boldsymbol{B}=\operatorname{im} u_{2}$. By Corollary 3.4, $\boldsymbol{K}_{0}$ and $\boldsymbol{B}$ are contained in $\boldsymbol{X}_{0}$. Thus the exact sequence $0-\rightarrow \boldsymbol{B} \rightarrow \boldsymbol{K}_{1} \rightarrow \rightarrow \operatorname{Tor}_{1}^{\Lambda}(M, \boldsymbol{T})-\rightarrow 0$ shows that $\operatorname{Ext}^{1}\left(\boldsymbol{T}, \boldsymbol{K}_{1}\right) \tilde{=} \operatorname{Ext}^{1}\left(\boldsymbol{T}, \operatorname{Tor}_{1}^{\Lambda}(M, \boldsymbol{T})\right)$. Application of $\operatorname{Hom}(\boldsymbol{T},-)$ to the exact sequences

$$
\begin{aligned}
& 0-\rightarrow K_{1}-\rightarrow P_{1} \rtimes_{\lambda} T-\rightarrow K_{0}-\rightarrow 0, \\
& 0-\rightarrow K_{0}-\rightarrow P_{0 \rtimes a} T-\rightarrow M \rtimes_{\lambda} T-\rightarrow 0
\end{aligned}
$$

leads to the commutative diagram with exact rows

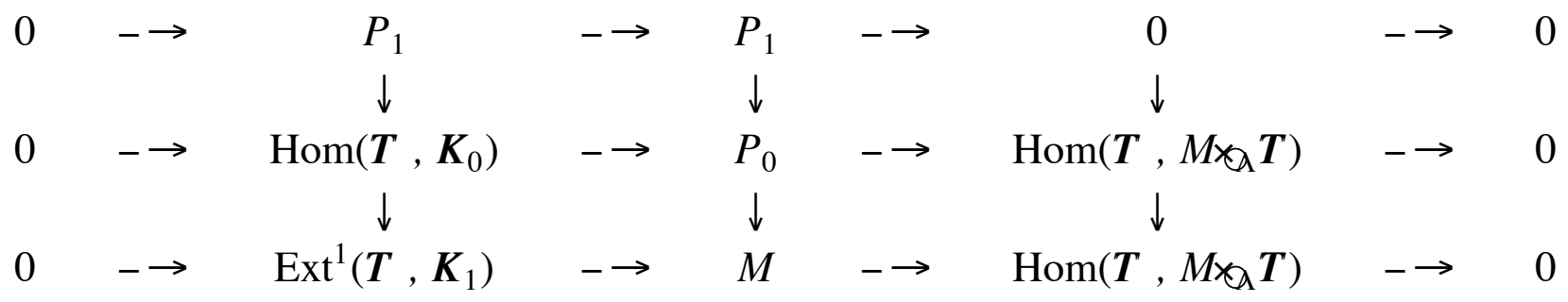
and the assertion follows.

Using this Lemma, we obtain:

3.8. Corollary. $\left(\boldsymbol{Y}_{1}, \boldsymbol{Y}_{0}\right)$ is a torsion theory for $\bmod \left(\Lambda^{o p}\right)$.

3.9. Corollary. $\operatorname{Ext}^{i}\left(\boldsymbol{T}, \operatorname{Tor}_{j}^{\Lambda}(M, \boldsymbol{T})\right)=0$ for all $i, j=0,1, i \neq j$ and all modules $M \in \bmod \left(\Lambda^{o p}\right)$.

From the proof of Theorem 3.3 and the fact that $\operatorname{Ext}_{A}^{i}(X, Y)=\operatorname{Hom}_{D^{b}(A)}\left(X, T^{i} Y\right)$ for all $X, Y$ in $\boldsymbol{A}$ where $\boldsymbol{A}=\operatorname{coh}(\boldsymbol{C})$ or $\boldsymbol{A}=\bmod \left(\Lambda^{o p}\right)$, we conclude

$$
\operatorname{Ext}_{\Lambda}^{l}\left(\operatorname{Ext}^{i}\left(\boldsymbol{T}, \boldsymbol{F}_{i}\right), \operatorname{Ext}^{j}\left(\boldsymbol{T}, \boldsymbol{F}_{j}\right)\right) \tilde{=} \operatorname{Ext}^{l-i+j}\left(\boldsymbol{F}_{i}, \boldsymbol{F}_{j}\right)
$$


for all $i, j, l$ and all $\boldsymbol{F}_{i} \in \boldsymbol{X}_{i}, \boldsymbol{F} \in \boldsymbol{X}_{j}$. This formula yields the following consequences:

3.10. Corollary. The torsion theory $\left(\boldsymbol{Y}_{1}, \boldsymbol{Y}_{0}\right)$ is splitting, i.e. each indecomposable module $M \in \bmod \left(\Lambda^{o p}\right)$ is either in $\boldsymbol{Y}_{0}$ or in $\boldsymbol{Y}_{1}$.

3.11. Corollary. gl.dim $\Lambda \leq 2$.

We denote by $K_{0}\left(\Lambda^{o p}\right)$ the Grothendieck group of $\bmod \left(\Lambda^{o p}\right)$. We get:

\subsection{Corollary.}

$$
f: K_{0}(\boldsymbol{C})-\rightarrow K_{0}\left(\Lambda^{o p}\right),[\boldsymbol{F}]-\rightarrow[\operatorname{Hom}(\boldsymbol{T}, \boldsymbol{F})]-\left[\operatorname{Ext}^{1}(\boldsymbol{T}, \boldsymbol{F})\right]
$$

is an isomorphism with inverse

$$
f^{-1}: K_{0}\left(\Lambda^{o p}\right)-\rightarrow K_{0}(C),[M]-\rightarrow[M \rtimes \boldsymbol{T}]-\left[\operatorname{Tor}_{1}^{\Lambda}(M, T)\right]
$$

\section{Sheaves and modules over canonical algebras}

In [30] Ringel introduced the class of canonical algebras, which might be defined as follows. Given $(\boldsymbol{p}, \boldsymbol{\lambda})$, we consider the quiver

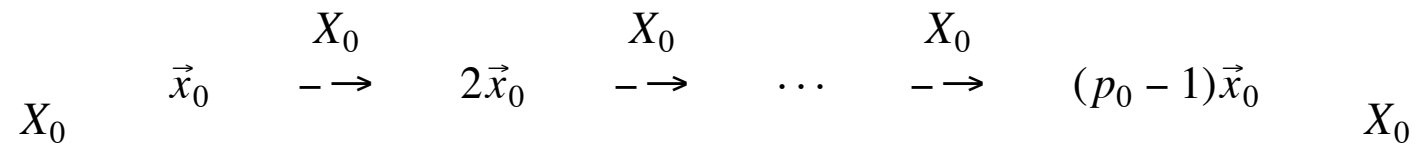

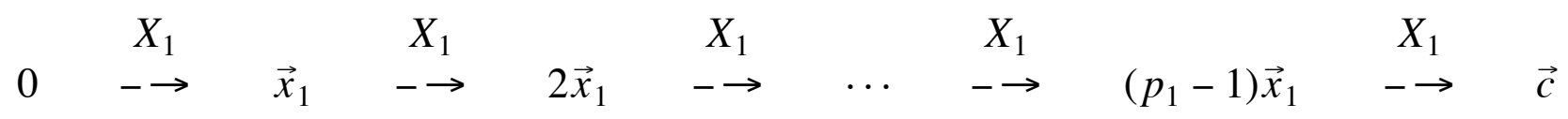

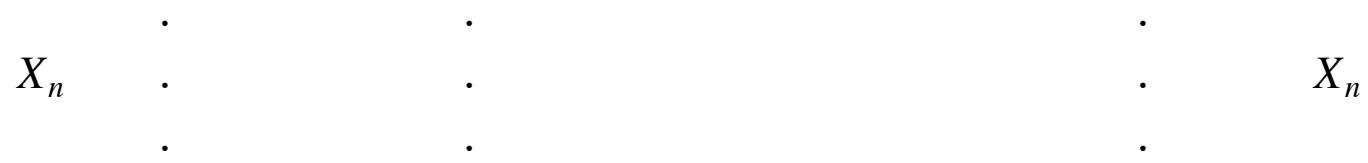

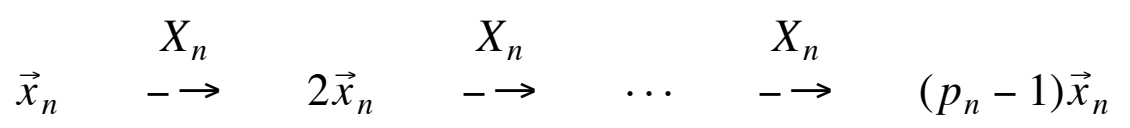

with relations given by

$$
X_{i}^{p_{i}}=X_{0}^{p_{0}}-\lambda_{i} X_{1}^{p_{1}} \quad \text { for } i=2, \ldots, n .
$$

Let $T=\bigoplus_{0 \leq \vec{x} \leq \vec{c}} \boldsymbol{O}_{C}(\vec{x})$ and $\Lambda=\operatorname{End}(\boldsymbol{T})$. By construction the full subcategory of $\operatorname{coh}(\boldsymbol{C})$, consisting of all $\boldsymbol{O}_{C}(\vec{x})$ for $0 \leq \vec{x} \leq \vec{c}$ is equivalent (so $\Lambda$ is Morita equivalent) to the path 
algebra of this quiver with respect to the relations (4.1.1). The canonical configuration $\boldsymbol{O}_{C}(\vec{x}), 0 \leq \vec{x} \leq \vec{c}$ visualizes the quiver in $\operatorname{coh}(\boldsymbol{C})$.

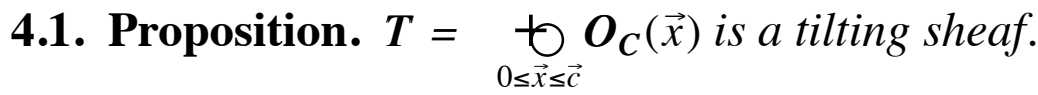

Proof: (1) Let $0 \leq \vec{x}, \vec{y} \leq \vec{c}$. By Serre duality we have

$$
\operatorname{DExt}^{1}\left(\boldsymbol{O}_{\boldsymbol{C}}(\vec{x}), \boldsymbol{O}_{\boldsymbol{C}}(\vec{y})\right) \tilde{=} \operatorname{Hom}\left(\boldsymbol{O}_{\boldsymbol{C}}(\vec{y}), \boldsymbol{O}_{\boldsymbol{C}}(\vec{x}+\vec{\omega})\right) .
$$

Since $\vec{\omega}+\vec{x}-\vec{y} \leq \vec{\omega}+\vec{c}$ is not positive, $\operatorname{Hom}\left(\boldsymbol{O}_{C}(\vec{y}), \boldsymbol{O}_{C}(\vec{x}+\vec{\omega})\right)=0$ follows. This proves $\operatorname{Ext}^{1}(\boldsymbol{T}, \boldsymbol{T})=0$.

(2) In order to prove that $\boldsymbol{T}$ generates $\mathrm{D}^{b}(\operatorname{coh}(\boldsymbol{C}))$ it is sufficient to show that $\operatorname{coh}(\boldsymbol{C})$ is the smallest subcategory $\boldsymbol{A}$ of $\operatorname{coh}(\boldsymbol{C})$, which contains all direct factors of $\boldsymbol{T}$, and is closed under the formation of kernels of epimorphisms, of cokernels of monomorphisms as well as under extensions. By means of the exact sequence (2.5.2) all exceptional simple sheaves are in $\boldsymbol{A}$. Since $\boldsymbol{O}_{C}$ is in $\boldsymbol{A}$, we conclude from (2.8.3) that all $\boldsymbol{O}_{C}(\vec{x})$ hence all vector bundles and all simple sheaves are in $A$.

(3) The quiver (4.1.1) has no oriented cycles, thus $\Lambda$ has finite global dimension. In fact $\operatorname{gl} \cdot \operatorname{dim} \Lambda \leq 2$.

The category $\boldsymbol{X}_{0}$ consists of all coherent sheaves with $\mathrm{H}^{1}(\boldsymbol{C}, \boldsymbol{F}(\vec{x}))=0$ for $-\vec{c} \leq \vec{x} \leq 0$; the category $X_{1}$ consists of all coherent sheaves with $\Gamma(\boldsymbol{C}, \boldsymbol{F}(\vec{x}))=0$ for $-\vec{c} \leq \vec{x} \leq 0$. We choose the following notation:

$$
\begin{gathered}
\operatorname{coh}^{+}(\boldsymbol{C}):=\boldsymbol{X}_{0}, \operatorname{coh}^{-}(\boldsymbol{C}):=\boldsymbol{X}_{1} . \\
\Gamma_{\Lambda}(\boldsymbol{C},-):=\operatorname{Hom}(\boldsymbol{T},-): \operatorname{coh}^{+}(\boldsymbol{C})-\rightarrow \boldsymbol{Y}_{0} . \\
\mathrm{H}_{\Lambda}^{1}(\boldsymbol{C},-):=\operatorname{Ext}^{1}(\boldsymbol{T},-): \operatorname{coh}^{-}(\boldsymbol{C})-\rightarrow Y_{1} .
\end{gathered}
$$

We note that all sheaves of finite length are contained in $\operatorname{coh}^{+}(\Lambda)$. Hence all sheaves in $\operatorname{coh}^{-}(C)$ are locally free. Moreover $\boldsymbol{O}_{\boldsymbol{C}}(\vec{x}) \in \operatorname{coh}^{+}(\boldsymbol{C})$ for all $\vec{x} \in \mathbf{L}_{+}$and $\boldsymbol{O}_{\boldsymbol{C}}(\vec{x}) \in \operatorname{coh}^{-}(\boldsymbol{C})$ otherwise. This implies that for each $\boldsymbol{F}$ in $\operatorname{coh}(\boldsymbol{C})$ there exists an $\vec{x} \in \mathbf{L}(\boldsymbol{p})$ such that $\boldsymbol{F}(\vec{y}) \in \operatorname{coh}^{+}(\boldsymbol{C})$ for all $\vec{y} \geq \vec{x}$.

A right $\Lambda$-module $M$ may be viewed as a representation

$$
\left(M_{\vec{x}}, X_{i}: M_{k \vec{x}_{i}}-\rightarrow M_{(k-1) \vec{x}_{i}}\right)
$$

of the quiver dual to (4.1.1). We call $M$ monoform (epiform, respectively) if all the linear maps $X_{i}$ are monomorphisms (epimorphisms, respectively) but not all of them are isomorphisms. 
The rank of $M$ is defined by

$$
\mathrm{r}=\operatorname{dim}_{k} M_{0}-\operatorname{dim}_{k} M_{\vec{c}} .
$$

4.2. Lemma. $\mathrm{r}\left(\Gamma_{\Lambda}(\boldsymbol{C}, \boldsymbol{F})\right)=\mathrm{r}(\boldsymbol{F})$ for all $\boldsymbol{F} \in \operatorname{coh}^{+}(\boldsymbol{C})$ and $\mathrm{r}\left(\mathrm{H}_{\Lambda}^{1}(\boldsymbol{C}, \boldsymbol{F})\right)=-\mathrm{r}(\boldsymbol{F})$ for all $\boldsymbol{F} \in \operatorname{coh}^{-}(\boldsymbol{C})$. In particular, if $\boldsymbol{F} \in \operatorname{coh}^{+}(\boldsymbol{C})$ is locally free, $\left.\Gamma_{\Lambda}(\boldsymbol{C}, \boldsymbol{F})\right)$ is monoform, and if $\left.\boldsymbol{F} \in \operatorname{coh}^{-}(\boldsymbol{C}), \mathrm{H}_{\Lambda}^{1}(\boldsymbol{C}, \boldsymbol{F})\right)$ is epiform.

Proof: We give the proof only in case $\boldsymbol{F} \in \operatorname{coh}^{+}(\boldsymbol{C})$; the case $\boldsymbol{F} \in \operatorname{coh}^{-}(\boldsymbol{C})$ is similar. So let $\boldsymbol{F} \in \operatorname{coh}(\boldsymbol{C})$. From the exact sequence (2.5.2)

$$
\left.0-\rightarrow \boldsymbol{O}_{C}\left(k \vec{x}_{i}\right)-\rightarrow \boldsymbol{O}_{C}(k+1) \vec{x}_{i}\right)-\rightarrow \boldsymbol{S}_{i, k}-\rightarrow 0
$$

we obtain the sequence

$$
\begin{gathered}
0-\rightarrow \operatorname{Hom}\left(\boldsymbol{S}_{i, k}, \boldsymbol{F}\right)-\rightarrow \operatorname{Hom}\left(\boldsymbol{O}_{\boldsymbol{C}}(k+1) \vec{x}_{i}, \boldsymbol{F}\right)-\rightarrow \operatorname{Hom}\left(\boldsymbol{O}_{\boldsymbol{C}}\left(k \vec{x}_{i}\right), \boldsymbol{F}\right)-\rightarrow \\
-\rightarrow \operatorname{Ext}^{1}\left(\boldsymbol{S}_{i, k}, \boldsymbol{F}\right)-\rightarrow \operatorname{Ext}^{1}\left(\boldsymbol{O}_{\boldsymbol{C}}\left((k+1) \vec{x}_{i}\right), \boldsymbol{F}\right)-\rightarrow \operatorname{Ext}^{1}\left(\boldsymbol{O}_{\boldsymbol{C}}\left(k \vec{x}_{i}\right), \boldsymbol{F}\right)-\rightarrow 0 .
\end{gathered}
$$

Since $\boldsymbol{F} \in \operatorname{coh}^{+}(\boldsymbol{C}), \operatorname{Ext}^{1}\left(\boldsymbol{O}_{\boldsymbol{C}}(k+1) \vec{x}_{i}, \boldsymbol{F}\right) \tilde{=} \operatorname{Ext}^{1}\left(\boldsymbol{O}_{\boldsymbol{C}}\left(k \vec{x}_{i}\right), \boldsymbol{F}\right) \tilde{=} 0$.

Suppose, $\boldsymbol{F}=\boldsymbol{O}_{\boldsymbol{C}}(\vec{x})$ is a twisted structure sheaf. Then $\operatorname{Hom}\left(\boldsymbol{S}_{i, k}, \boldsymbol{O}_{\boldsymbol{C}}(\vec{x})\right)=0$ and $\operatorname{Ext}^{1}\left(S_{i, k}, O_{C}(\vec{x})\right) \neq 0 \quad$ only for one $k \in\left\{0, \ldots, p_{i}-1\right\}$. In this case $\operatorname{dim}_{k} \operatorname{Ext}^{1}\left(\boldsymbol{S}_{i, k}, \boldsymbol{O}_{\boldsymbol{C}}(\vec{x})\right)=1$ hence $\mathrm{r}\left(\Gamma_{\Lambda}\left(\boldsymbol{C}, \boldsymbol{O}_{\boldsymbol{C}}(\vec{x})\right)=1\right.$.

Now, assume that $\boldsymbol{F}$ is a sheaf of finite length, thus $\operatorname{Hom}\left(\boldsymbol{O}_{\boldsymbol{C}}(0), \boldsymbol{F}\right) \tilde{=} \operatorname{Hom}\left(\boldsymbol{O}_{\boldsymbol{C}}(\vec{c}), \boldsymbol{F}\right)$, hence $\mathrm{r}\left(\Gamma_{\Lambda}(\boldsymbol{C}, \boldsymbol{F})=0\right.$. Finally, suppose that $\boldsymbol{F}$ is locally free of rank $n$. There is an exact sequence

$$
0-\rightarrow \boldsymbol{F}-\rightarrow \underset{i=1}{n} \boldsymbol{O}_{C}\left(\vec{y}_{i}\right)-\rightarrow \boldsymbol{L}-\rightarrow 0
$$

with $\boldsymbol{L}$ of finite length (2.7). We get $\mathrm{r}\left(\Gamma_{\Lambda}(\boldsymbol{C}, \boldsymbol{F})\right)=\mathrm{r}\left(\Gamma_{\Lambda}\left(\boldsymbol{C}, \underset{i=1}{\operatorname{t}} \boldsymbol{O}_{\boldsymbol{C}}\left(\vec{y}_{i}\right)\right)=n=\mathrm{r}(\boldsymbol{F})\right.$. In particular, $\Gamma_{\Lambda}(\boldsymbol{C}, \boldsymbol{F})$ is monoform since $\operatorname{Hom}\left(\boldsymbol{S}_{i, k}, \boldsymbol{F}\right)=0$ for all $i, k$, and there exists some $\boldsymbol{S}_{i, k}$ with $\operatorname{Ext}^{1}\left(\boldsymbol{S}_{i, k}, \boldsymbol{F}\right) \neq 0$.

Let $\bmod ^{+}\left(\Lambda^{o p}\right)$ and $\bmod ^{-}\left(\Lambda^{o p}\right)$ be the full subcategories of $\bmod \left(\Lambda^{o p}\right)$ consisting of all modules $M$, such that each indecomposable direct factor is monoform (epiform, respectively). Let $\bmod ^{0}\left(\Lambda^{o p}\right)$ be the category of all modules, whose indecomposable direct factors are neither in $\bmod ^{+}\left(\Lambda^{o p}\right)$ nor in $\bmod ^{-}\left(\Lambda^{o p}\right)$.

From Lemma 4.2 we deduce that $\bmod ^{+}\left(\Lambda^{o p}\right)$ is equivalent to the category of locally free sheaves contained in $\operatorname{coh}^{+}(\boldsymbol{C})$ by means of functor $\Gamma_{\Lambda}(\boldsymbol{C},-)$. Further we have equivalences $\quad \mathrm{H}_{\Lambda}^{1}: \operatorname{coh}^{-}(\boldsymbol{C}) \rightarrow \bmod ^{-}\left(\Lambda^{o p}\right) \quad$ and $\quad \Gamma_{\Lambda}: \operatorname{coh}^{0}(\boldsymbol{C}) \rightarrow \bmod ^{0}\left(\Lambda^{o p}\right), \quad$ thus 
$\bmod ^{0}\left(\Lambda^{o p}\right) \tilde{=} \underset{x \in C}{\Perp} \bmod ^{0}\left(\Lambda^{o p}\right)_{x}$.

According to [30], we say that $\bmod ^{0}\left(\Lambda^{o p}\right)$ separates $\bmod ^{+}\left(\Lambda^{o p}\right)$ from $\bmod ^{-}\left(\Lambda^{o p}\right)$ if the following two conditions are satisfied:

(1) $\operatorname{Hom}_{\Lambda}(Z, X)=\operatorname{Hom}_{\Lambda}(Y, Z)=\operatorname{Hom}_{\Lambda}(Y, X)=0 \quad$ for all modules $X \in \bmod ^{+}\left(\Lambda^{o p}\right)$, $Y \in \bmod ^{0}\left(\Lambda^{o p}\right)$, and $Z \in \bmod ^{-}\left(\Lambda^{o p}\right)$.

(2) Each morphism $f: X-\rightarrow Z, X \in \bmod ^{+}\left(\Lambda^{o p}\right), Z \in \bmod ^{-}\left(\Lambda^{o p}\right)$, admits a factorization $f=[X-\rightarrow Y-\rightarrow Z]$ with $Y \in \bmod ^{0}\left(\Lambda^{o p}\right)$. Moreover, $Y$ may be chosen in a prescribed component $\bmod ^{0}\left(\Lambda^{o p}\right)_{x}$.

4.3. Proposition ( [30]). An indecomposable module $M$ is in $\bmod ^{+}\left(\Lambda^{o p}\right), \bmod ^{0}\left(\Lambda^{o p}\right)$ or $\bmod ^{-}\left(\Lambda^{o p}\right)$, if and only if $\mathrm{r}(M)>0, \mathrm{r}(M)=0, \mathrm{r}<0$, respectively. Moreover, $\bmod ^{0}\left(\Lambda^{o p}\right)$ separates $\bmod ^{+}\left(\Lambda^{o p}\right)$ from $\bmod ^{-}\left(\Lambda^{o p}\right)$.

Proof: The first assertion is covered by Lemma 4.2. Let $X \in \bmod ^{+}\left(\Lambda^{o p}\right), Y \in \bmod ^{0}\left(\Lambda^{o p}\right)$, and $Z \in \bmod ^{-}\left(\Lambda^{o p}\right)$. By means of Theorem 3.3 we have $\operatorname{Hom}_{\Lambda}(Y, X)=0$, since there are no non-zero morphisms from sheaves of finite length to locally free sheaves and $\operatorname{Hom}_{\Lambda}(Y, Z)=\operatorname{Hom}_{\Lambda}(X, Z)=0$ since $X, Y \in Y_{0}$ and $Z \in Y_{1}$.

Let $f: X \rightarrow Z$ be a morphism, $\boldsymbol{F}=X_{\rtimes_{\lambda}} \boldsymbol{T}$ and $\boldsymbol{G}=\operatorname{Tor}_{1}^{\Lambda}(Y, \boldsymbol{T})$. There exists an exact sequence $0-\rightarrow \boldsymbol{G} \rightarrow \boldsymbol{F}^{\prime} \rightarrow \boldsymbol{L} \rightarrow \rightarrow 0$, where $\boldsymbol{L}$ belongs to a fixed component $\operatorname{cof}_{0}(\boldsymbol{C})$ and such that $\operatorname{Ext}^{1}\left(\boldsymbol{F}, \boldsymbol{F}^{\prime}\right)=0$ (Corollary 2.7). From the exactness of

$$
0-\rightarrow \Gamma_{\Lambda}\left(\boldsymbol{C}, \boldsymbol{F}^{\prime}\right)-\rightarrow \Gamma_{\Lambda}(\boldsymbol{C}, \boldsymbol{L})-\rightarrow Z-\rightarrow 0
$$

we conclude that $f$ can be lifted to $\Gamma_{\Lambda}(\boldsymbol{C}, \boldsymbol{L})$ since $\operatorname{Ext}_{\Lambda}^{1}\left(X, \Gamma_{\Lambda}\left(\boldsymbol{C}, \boldsymbol{F}^{\prime}\right)\right)=0$.

In the the language of [30], Proposition 4.3 shows that ind ${ }^{0}\left(\Lambda^{o p}\right)$, the category of all indecomposable modules in $\bmod ^{0}\left(\Lambda^{o p}\right)$, is a separating tubular family of type $\left(p_{0}, \ldots, p_{n}\right)$.

The categories $\operatorname{coh}(C)$ and $\bmod \left(\Lambda^{o p}\right)$ have almost-split sequences. Typically almostsplit sequences in $\operatorname{coh}(\boldsymbol{C})$ give rise to almost-split sequences in $\bmod \left(\Lambda^{o p}\right)$ :

4.4. Proposition. Let $0-\rightarrow \boldsymbol{F} \rightarrow \rightarrow \boldsymbol{M} \rightarrow \boldsymbol{G}-\rightarrow 0$ be an almost-split sequence in $\operatorname{coh}(\boldsymbol{C})$. If $\boldsymbol{F}$ and $\boldsymbol{G}$ are in $\operatorname{coh}^{+}(\boldsymbol{C})$,

$$
0-\rightarrow \Gamma_{\Lambda}(\boldsymbol{C}, \boldsymbol{F})-\rightarrow \Gamma_{\Lambda}(\boldsymbol{C}, \boldsymbol{M})-\rightarrow \Gamma_{\Lambda}(\boldsymbol{C}, \boldsymbol{G})-\rightarrow 0
$$

is an almost-split sequence in $\bmod \left(\Lambda^{o p}\right)$; if $\boldsymbol{F}$ and $\boldsymbol{G}$ are in $\operatorname{coh}^{-}(\boldsymbol{C})$,

$$
0-\rightarrow \mathrm{H}_{\Lambda}^{1}(\boldsymbol{C}, \boldsymbol{F})-\rightarrow \mathrm{H}_{\Lambda}^{1}(\boldsymbol{C}, \boldsymbol{M})-\rightarrow \mathrm{H}_{\Lambda}^{1}(\boldsymbol{C}, \boldsymbol{G})-\rightarrow 0
$$

is an almost-split sequence in $\bmod \left(\Lambda^{o p}\right)$. 
We note that the categories $\bmod ^{+}\left(\Lambda^{o p}\right), \bmod ^{0}\left(\Lambda^{o p}\right)$, and $\bmod ^{-}\left(\Lambda^{o p}\right)$ are stable under the Auslander-Reiten translation.

Let $\boldsymbol{F}$ be an indecomposable coherent sheaf not contained in $\operatorname{coh}^{+}(\boldsymbol{C})$ and $\operatorname{coh}^{-}(\boldsymbol{C})$, and let $0-\rightarrow \boldsymbol{F}_{+} \rightarrow \boldsymbol{F}-\rightarrow \boldsymbol{F}_{-} \rightarrow 0$ be exact with $\boldsymbol{F}_{+} \in \operatorname{coh}^{+}(\boldsymbol{C})$ and $\boldsymbol{F}_{-} \in \operatorname{coh}^{-}(\boldsymbol{C})$. From formula (3.10.1) we derive $\operatorname{Ext}^{1}\left(\boldsymbol{F}_{-}, \boldsymbol{F}_{+}\right)=\operatorname{Ext}_{\Lambda}^{2}\left(\mathrm{H}_{\Lambda}^{1}\left(\boldsymbol{C}, \boldsymbol{F}_{-}\right), \Gamma_{\Lambda}\left(\boldsymbol{C}, \boldsymbol{F}_{+}\right)\right)$, hence each indecomposable direct factor of $\Gamma_{\Lambda}\left(\boldsymbol{C}, \boldsymbol{F}_{+}\right)$has injective dimension two and each indecomposable direct factor of $\mathrm{H}_{\Lambda}^{1}\left(\boldsymbol{C}, \boldsymbol{F}_{-}\right)$has projective dimension two. Conversely, if $\boldsymbol{G} \in \operatorname{coh}^{+}(\boldsymbol{C})$ is indecomposable and $\Gamma_{\Lambda}(\boldsymbol{C}, \boldsymbol{G})$ has injective dimension two, there exist $\boldsymbol{F}_{-} \in \operatorname{coh}^{-}(\boldsymbol{C})$ and an exact sequence $0-\rightarrow \boldsymbol{G} \rightarrow \boldsymbol{F}-\rightarrow \boldsymbol{F}_{-} \rightarrow 0$; if $\boldsymbol{G} \in \operatorname{coh}^{-}(\boldsymbol{C})$ is indecomposable and $\mathrm{H}_{\Lambda}^{1}(\boldsymbol{C}, \boldsymbol{G})$ has projective dimension two, there exists $\boldsymbol{F}_{+} \in \operatorname{coh}^{+}(\boldsymbol{C})$ and an exact sequence $0-\rightarrow \boldsymbol{F}_{+}-\rightarrow \boldsymbol{F} \rightarrow \boldsymbol{G} \rightarrow \rightarrow 0$.

4.5. Proposition. (1) Let $M \in \bmod ^{+}\left(\Lambda^{o p}\right)$ or $M \in \bmod ^{0}\left(\Lambda^{o p}\right)$ be indecomposable and $0 \rightarrow M-B \rightarrow N \rightarrow 0$ be an almost-split sequence in $\bmod \left(\Lambda^{o p}\right)$. Then $0-\rightarrow M \rtimes_{\lambda} \boldsymbol{T} \rightarrow E_{\searrow} \boldsymbol{T} \rightarrow N_{\lambda} \boldsymbol{T}-\rightarrow 0$ is an almost-split sequence in $\operatorname{coh}(\boldsymbol{C})$ if and only if inj $\operatorname{dim} M \leq 1$.

(2) Let $M \in \bmod ^{-}\left(\Lambda^{o p}\right)$ be indecomposable and $0-\rightarrow N \rightarrow E \rightarrow M-\rightarrow 0$ be an almost-split sequence in $\bmod \left(\Lambda^{o p}\right)$. Then $0-\rightarrow \operatorname{Tor}_{1}^{\Lambda}(N, \boldsymbol{T}) \rightarrow \operatorname{Tor}_{1}^{\Lambda}(E, \boldsymbol{T})-\rightarrow \operatorname{Tor}_{1}^{\Lambda}(M, \boldsymbol{T})-\rightarrow 0$ is an almost-split sequence if and only if proj $\operatorname{dim} M \leq 1$.

Proof: We give only a proof for (1), since the proof for (2) is dual. Thus let $M \in \bmod ^{+}\left(\Lambda^{o p}\right)$ or $M \in \bmod ^{0}\left(\Lambda^{o p}\right)$ be indecomposable. Since $M$ is not injective, an almost-split sequence $0 \rightarrow M-\rightarrow E \rightarrow N \rightarrow 0$ exists. Suppose that the injective dimension of $M$ is one, and $f: M \rtimes_{a} \boldsymbol{T} \rightarrow \boldsymbol{F}$ is a non-zero morphism where $\boldsymbol{F}$ is indecomposable and $f$ is not an isomorphism. We have to show that $f$ extends to $E x \boldsymbol{T}$. If $\boldsymbol{F} \in \operatorname{coh}^{+}(\boldsymbol{C})$ we are done. Otherwise, $\boldsymbol{F}$ is neither in $\operatorname{coh}^{+}(\boldsymbol{C})$ nor on $\operatorname{coh}^{-}(\boldsymbol{C})$. Thus there exists an exact sequence $0-\rightarrow \boldsymbol{F}_{+} \rightarrow \boldsymbol{F}-\rightarrow \boldsymbol{F}_{-} \rightarrow 0$ with $\boldsymbol{F}_{+} \in \operatorname{coh}^{+}(\boldsymbol{C})$ and $\boldsymbol{F}_{-} \in \operatorname{coh}^{-}(\boldsymbol{C})$. Since $\operatorname{Hom}\left(M \star_{\alpha} \boldsymbol{T}, \boldsymbol{F}_{-}\right)=0$, the morphism $f$ factors through $\boldsymbol{F}_{+}$. The morphism $M \rtimes \boldsymbol{T} \rightarrow \boldsymbol{F}_{+}$is not a splittable monomorphism, since all direct summands of $\Gamma_{\Lambda}\left(\boldsymbol{C}, \boldsymbol{F}_{+}\right)$have injective dimension two. Thus this morphism, hence also $f$, can be extended to $E_{\rtimes} T$.

Now suppose that inj $\operatorname{dim} M=2$ and that $0 \rightarrow M \rtimes_{\lambda} \boldsymbol{T} \rightarrow E \rtimes_{\lambda} \boldsymbol{T} \rightarrow N_{\lambda} \boldsymbol{T}-\rightarrow 0$ is an almost-split sequence. Since inj $\operatorname{dim} M=2$, there exist a non-split exact sequence $0-\rightarrow M \rtimes_{\lambda} \boldsymbol{T} \rightarrow \boldsymbol{F} \rightarrow \rightarrow \boldsymbol{F}_{-}-\rightarrow 0$ with $\boldsymbol{F}_{-} \in \operatorname{coh}^{-}(\boldsymbol{C})$. The extension property of almostsplit sequences leads to a non-zero morphism $N \rtimes_{a} \boldsymbol{T} \rightarrow \boldsymbol{F}_{-}$, a contradiction. 


\section{Classification for bundles and modules}

Let $S=S(\boldsymbol{p}, \lambda)$, we consider the polynomial algebra $R=k\left[x_{0}^{p_{0}}, x_{1}^{p_{1}}\right]$ now as an $\mathbf{L}(\boldsymbol{p})$-graded subalgebra. As follows from (1.3.3), the elements

$$
x_{0}^{l_{0}} \cdots x_{n}^{l_{n}} \quad\left(0 \leq l_{i}<p_{i}\right)
$$

form an $\mathbf{L}(\boldsymbol{p})$-homogeneous basis for $S$ over $R$, so $S$ is an $\mathbf{L}(\boldsymbol{p})$-graded Cohen-Macaulay algebra. We denote by

$$
\mathrm{CM}^{\mathbf{L}(p)}(S)
$$

the category of all $M \in \bmod ^{\mathbf{L}(p)}(S)$, which are finitely generated free as $\mathbf{L}(\boldsymbol{p})$-graded $R$ modules. By definition these are the (maximal) $\mathbf{L}(p)$-graded Cohen-Macaulay modules over $S$. Note that all $S(\vec{x}), \vec{x} \in \mathbf{L}(p)$ are in $\operatorname{CM}^{\mathbf{L}(p)}(S)$.

We are now going to prove a refinement of Serre's theorem 1.8; again $\boldsymbol{C}=\boldsymbol{C}(\boldsymbol{p}, \lambda)$.

5.1. Theorem. The $\mathbf{L}(\boldsymbol{p})$-graded global sections functor induces an equivalence $\Gamma: \operatorname{vect}(\boldsymbol{C})-\rightarrow \mathrm{CM}^{\mathbf{L}(\boldsymbol{p})}(S)$. Moreover, $\mathrm{CM}^{\mathbf{L}(p)}(S)$ consists of all $M \in \bmod ^{\mathbf{L}(p)}(S)$, where $\operatorname{Hom}(E, M)=0=\operatorname{Ext}^{1}(E, M)$ holds for each simple $\mathbf{L}(\boldsymbol{p})$-graded $S$-module $E$.

We note that (5.1) establishes a link to the study of Cohen-Macaulay modules for the isolated singularity 0 of the surface $\boldsymbol{F}(\boldsymbol{p}, \lambda)$, see for instance [4].

Proof. We denote by $\boldsymbol{A}(S)$ the category consisting of all $M \in \bmod ^{\mathbf{L}(p)}(S)$, satisfying $\operatorname{Hom}(E, M)=0=\operatorname{Ext}^{1}(E, M)$ for all simple graded modules $E$. Since $S$ is noetherian, by means of (1.7.1) using a line bundle filtration for $\boldsymbol{F}$, we conclude that $\Gamma(F)$ is finitely generated over $S$. Moreover $\Gamma(F) \in A(S)$, as one may deduce from Serre's theorem. Conversely, if $M \in A(S), \tilde{M}$ is a vector bundle over $C$ : First note that $M=(\Gamma(M))^{\sim}$, since $M \in \boldsymbol{A}(S)$, using Serre's theorem for quasicoherent sheaves in combination with ( [12], p. 372). If $\tilde{M}$ has a simple subsheaf $\boldsymbol{E}, M$ contains $\Gamma(\boldsymbol{E})=U$ as a submodule. Since $U_{\vec{x}} \neq 0$ for infinitely many $\vec{x} \leq 0, U$ hence $M$ is not finitely generated, a contradiction. We conclude that

$$
\Gamma: \operatorname{vect}(\boldsymbol{C})-\rightarrow \mathrm{CM}^{\mathbf{L}(p)}(S) \text { and } \sim \operatorname{CM}^{\mathbf{L}(\boldsymbol{p})}(S)-\rightarrow \operatorname{vect}(\boldsymbol{C})
$$

define mutually inverse equivalences of categories.

Passing to the special case $\boldsymbol{C}=\mathbf{P}_{1}(k)$, we see that $\operatorname{vect}\left(\mathbf{P}_{1}(k)\right)$ and $\boldsymbol{A}(R)$ are equivalent categories. We infer from (1.7.1) in combination with Grothendieck's theorem [16] that $\boldsymbol{A}(R)$ consists just of all free modules in $\bmod ^{\mathbf{L}(\boldsymbol{p})}(R)$. 
It thus remains to prove for $M \in \bmod ^{\mathbf{L}(\boldsymbol{p})}(S)$ that $M \in \boldsymbol{A}(S)$ if and only $M \in \boldsymbol{A}(R)$. Let

$$
0-\rightarrow S(-2 \vec{c})-\stackrel{\alpha}{\rightarrow} S(-\vec{c}) \text { to } S(-\vec{c})-\stackrel{\beta}{\rightarrow} S-\rightarrow E-\rightarrow 0
$$

with $\alpha=\left(x_{0}^{p_{0}}, x_{1}^{p_{1}}\right)$ and $\beta=\left(x_{1}^{p_{1}},-x_{0}^{p_{0}}\right)$ be the Koszul complex given by the regular sequence $x_{0}^{p_{0}}, x_{1}^{p_{1}}$ in $S$. It is easily checked that $M \in A(S)$ if and only if $\operatorname{Hom}_{S}(E(\vec{x}), M)=0=\operatorname{Ext}_{S}^{1}(E(\vec{x}), M)$ for each $\vec{x} \in \mathbf{L}(\boldsymbol{p})$. An equivalent assertion is the exactness of

$$
0-\rightarrow M_{\vec{x}}-\rightarrow M_{\vec{x}-\vec{c}} † M_{\vec{x}-\vec{c}}-\rightarrow M_{\vec{x}-2 \vec{c}}
$$

for each $\vec{x} \in \mathbf{L}(\boldsymbol{p})$. Let $\mathrm{V} \subset \mathbf{L}(\boldsymbol{p})$ consist of all $\sum_{i=0}^{n} l_{i} \vec{x}_{i}$, with $0 \leq l_{i}<p_{i}$. If $k=R / R_{+}$, we have $E=\bigoplus_{\vec{x} \in \mathrm{V}} k(\vec{x})$ as graded $R$-modules. Accordingly $M \in A(R)$ if and only if $\operatorname{Hom}_{R}(E(\vec{x}), M)=0=\operatorname{Ext}_{R}^{1}(E(\vec{x}), M)$. By means of (5.1.1) this amounts to exactness of

$$
0-\rightarrow \bigoplus_{\vec{v} \in \mathrm{V}} M_{\vec{x}}(\vec{v})-\rightarrow \bigoplus_{\vec{v} \in \mathrm{V}}\left(M_{\vec{x}-\vec{c}}(\vec{v}) \text { † } M_{\vec{x}-\vec{c}}(\vec{v})\right)-\rightarrow \bigoplus_{\vec{v} \in \mathrm{V}} M_{\vec{x}-2 \vec{c}}(\vec{v}),
$$

an assertion equivalent to exactness of all sequences (5.1.2).

For each non-zero vector bundle let $\mu(F)=\frac{\mathrm{d}(F)}{\mathrm{r}(F)}$, where $\mathrm{d}$ and $\mathrm{r}$ denote rank and degree, respectively. $F$ is called semi-stable (stable) if for each non-zero sub-bundle $F^{\prime}$ of $F$ we have $\mu\left(F^{\prime}\right) \leq \mu(F)\left(\mu\left(F^{\prime}\right)<\mu(F)\right.$, respectively $)$.

As in the case of smooth projective curves we have the following result due to Narasimhan and Seshadri [32]:

5.2. Proposition. For each $q \in \mathbf{Q}$ let $\boldsymbol{C}_{q}$ denote the category consisting of the zero bundle and all semi-stable vector bundles $F$ with $\mu(F)=q$. The following properties hold true:

(i) Each $C_{q}$ is an exact abelian subcategory of $\operatorname{coh}(\boldsymbol{C})$, closed under extensions.

(ii) Each $F \in C_{q}$ has finite length in $\boldsymbol{C}_{q}$. The simple objects in $\boldsymbol{C}_{q}$ are just the stable bundles; in particular $\operatorname{End}(F)=k$ if $F$ is stable.

(iii) If $F \in \boldsymbol{C}_{q}, F^{\prime} \in \boldsymbol{C}_{q}{ }^{\prime}$ and $\operatorname{Hom}\left(F, F^{\prime}\right) \neq 0$ then $q \leq q^{\prime}$.

Proof: (i) is easily checked. (ii) Assume $0 \neq F^{\prime} \subset F$ are both in $C_{q}$ with $\mathrm{r}\left(F^{\prime}\right)=\mathrm{r}(F)$, hence $\mathrm{d}\left(F^{\prime}\right)=\mathrm{d}(F)$. Since $\mathrm{r}\left(F / F^{\prime}\right)=0$, the sheaf $F / F^{\prime}$ has finite length, from $\mathrm{d}\left(F / F^{\prime}\right)=0$ we conclude $F / F^{\prime}=0$. Thus any chain of subobjects of $F$ within $C_{q}$ has length $\leq \mathrm{r}(F)$. To prove (iii) note that $\mu\left(F / F^{\prime}\right) \geq \mu(F)$ for each semi-stable $F$. 
As in the case of smooth projective curves (cf. [32]) bundles on $\boldsymbol{C}$ have a HarderNarasimhan filtration as follows from

5.3. Lemma. Each non-zero bundle $F$ on $\boldsymbol{C}$ has a non-zero sub-bundle $F_{1}$ such that each non-zero sub-bundle (sub-sheaf) $F^{\prime}$ of $F$ satisfies $\mu\left(F^{\prime}\right) \leq \mu\left(F_{1}\right) . F_{1}$ is uniquely determined if we assume additionally that $F_{1}$ has maximal rank.

In particular $F_{1}$ is semi-stable, called the maximal semi-stable sub-bundle of $F$.

Proof. If $0=F_{0} \subset F_{1} \subset \cdots \subset F_{n}=F$ is a line bundle filtration for $F$, each non-zero $F^{\prime} \subset F$ satisfies

$$
\mu\left(F^{\prime}\right) \leq \sum_{i=1}^{n}\left|\mathrm{~d}\left(F_{i} / F_{i-1}\right)\right|
$$

Now choose $0 \neq F_{1} \subset F$ with $\mu\left(F_{1}\right)$ being maximal.

5.4. Remark. The complexity of the classification problem for $\operatorname{coh}(\boldsymbol{C})$, hence for $\operatorname{vect}(\boldsymbol{C})$, depends mainly on the virtual genus of $C$, equivalently on the degree $\delta(\vec{\omega})$ of the dualizing sheaf $\boldsymbol{O}_{C}(\vec{\omega})$. We have to distinguish the following cases:

5.4.1. If $\delta(\vec{\omega})<0$ (accordingly $g_{v}(C)<1$ ), we deal with the weight sequence attached to a Dynkin diagram $\Delta=\mathbf{A}_{p, q}(p \geq 1, q \geq 1), \mathbf{D}_{n}(n \geq 4), \mathbf{E}_{6}, \mathbf{E}_{7}, \mathbf{E}_{8}$, by counting the length of the arms of $\Delta$. If $n=1$, i.e. $\Delta=\mathbf{A}_{p, q}$, we just deal with the weighted projective line $\mathbf{P}_{1}(p, q)$; if $n=2$, i.e. $\Delta=\mathbf{D}_{n}$ or $\mathbf{E}_{6}, \mathbf{E}_{7}, \mathbf{E}_{8}, \boldsymbol{C}$ is defined by just one equation

$$
X_{2}^{p_{2}}-X_{1}^{p_{1}}+X_{0}^{p_{0}}=0 \text {, }
$$

and no parameters $\lambda_{i}$ occur.

In this situation the canonical algebra $\Lambda=\Lambda\left(p_{0}, p_{1}, p_{2}\right)$ arises as a tilted algebra of a tame hereditary algebra $\Sigma$ of extended Dynkin type $\tilde{\Delta}$, actually as a so-called concealed quiver algebra. [30]. By means of the comparison theorem we get $\mathrm{D}^{b}(\bmod (\Sigma))=\mathrm{D}^{b}(\operatorname{coh}(\boldsymbol{C}))$, hence the classification for $\operatorname{coh}(\boldsymbol{C})$ is equivalent to the classification for $\bmod (\Sigma)$. We refer to [26] for the details of the transfer of the classification for $\bmod (\Sigma)$, due to Nazarova [28] and Donovan-Freislich [11] (see also [9]) to the classification for $\operatorname{coh}(C)$.

Suppose now $k=\mathbf{C}$. Let $G \subset \operatorname{SL}(2, \mathbf{C})$ be a binary polyhedral group of Dynkin type $\Delta=\left(p_{0}, p_{1}, p_{2}\right)$, i.e. $G$ has generators $\xi_{0}, \xi_{1}, \xi_{2}$ and relations $\xi_{0}^{p_{0}}=\xi_{1}^{p_{1}}=\xi_{2}^{p_{2}}=\xi_{0} \xi_{1} \xi_{2}$. The algebra of relative invariants $A^{G \text {,rel }}$ with respect to the natural action of $G$ on $A=k\left[T_{0}, T_{1}\right]$ is generated by three fundamental relative invariants $F_{0}, F_{1}, F_{2}$, subject to 
relation (5.4.1). As was proved already by F. Klein [25]

$$
k\left[X_{0}, X_{1}, X_{2}\right] /\left(X_{2}^{p_{2}}-X_{1}^{p_{1}}+X_{0}^{p_{0}}\right) \tilde{=} A^{G, r e l} .
$$

We refer to the survey of Slodowy [33] for further information.

$A^{G . r e l}$ is naturally $G^{*} \times \mathbf{Z}$-graded, where $G^{*}$ denotes the character group of $G$. Moreover $\mathbf{L}(p)$ may be identified with a subgroup of $G^{*} \times \mathbf{Z}$ attaching to $\vec{x}_{i}$ the pair $(\chi, n)$ where $F_{i}$ has degree $n$ in $A$ and weight $\chi \in G^{*}$. So we obtain $S(\boldsymbol{p}, \lambda)$ by restriction of $A^{G, r e l}$ to the subgroup $\mathbf{L}(\boldsymbol{p})$ of $G^{*} \times \mathbf{Z}$.

5.4.2. $\delta(\vec{\omega})=0$, accordingly $g_{v}(\boldsymbol{C})=1$. Here we deal with the cases $(2,2,2,2),(3,3,3)$, $(2,4,4),(2,3,6)$ - called tubular by Ringel [30] - corresponding to the extended Dynkin diagrams $\tilde{\mathbf{D}}_{4}, \tilde{\mathbf{E}}_{6}, \tilde{\mathbf{E}}_{7}$ and $\tilde{\mathbf{E}}_{8}$, respectively. By means of the comparison $\mathrm{D}^{b}(\operatorname{coh}(\boldsymbol{C}))=\mathrm{D}^{b}(\bmod (\Lambda))$, classification for $\operatorname{coh}(\boldsymbol{C})$ reduces to the classification for $\bmod (\Lambda)$, with $\Lambda$ a canonical algebra of tubular type [30] and conversely. In the rest of this section we will show that the classification for $\operatorname{coh}(\boldsymbol{C})$ is possible along the lines of Atiyah's classification for vector bundles ( [1] , see also [29] ) on smooth elliptic curves. This will relate Atiyah's classification with Ringel's classification [30] for modules over tubular algebras.

5.4.3. $\delta(\vec{\omega})>0$, accordingly $g_{v}(\boldsymbol{C})>1$. Here, the corresponding canonical algebras $\Lambda$ are wild. By comparison $\mathrm{D}^{b}(\operatorname{coh}(\boldsymbol{C}))=\mathrm{D}^{b}(\bmod (\Lambda))$, classification for $\operatorname{coh}(\boldsymbol{C})$ is also a wild problem. The treatment of smooth projective curves by Narasimhan and Seshadri (compare [32]) suggests to develop a classification of stable bundles by means of moduli spaces also for $\operatorname{coh}(\boldsymbol{C})$.

We note that in all three cases it is easy to determine the structure of AuslanderReiten components using [19].

By means of the Harder-Narasimhan filtration it is easy to deal with the cases $\delta(\vec{\omega}) \leq 0$ :

5.5. Proposition. Let $F$ be an indecomposable vector bundle on $\boldsymbol{C}=\boldsymbol{C}(\boldsymbol{p}, \lambda)$ :

(i) If $\delta(\vec{\omega})<0$, correspondingly $\Lambda(\boldsymbol{p}, \lambda)$ is a concealed tame quiver algebra, $F$ is stable and $\operatorname{End}(F)=k$.

(ii) If $\delta(\vec{\omega})=0$, accordingly $\Lambda(\boldsymbol{p}, \lambda)$ is a tubular algebra, $F$ is semi-stable. 
Proof: Let $\delta(\vec{\omega}) \leq 0$. Consider an exact sequence

$$
\eta: 0 \rightarrow F_{1} \rightarrow F-\rightarrow F / F_{1} \rightarrow 0,
$$

where $F_{1}$ is the maximal semi-stable sub-bundle of $F$. Assume $F / F_{1} \neq 0$, so $\eta$ does not split since $F$ is indecomposable. By Serre duality we get a non-zero $u: F_{1}(-\vec{\omega})-\rightarrow\left(F / F_{1}\right)$. Since $\delta(\vec{\omega}) \leq 0$, we have $\mu\left(F_{1}(-\vec{\omega})\right) \geq \mu\left(F_{1}\right)$. Hence $F / F_{1}$ has a non-zero subsheaf $F_{2} / F_{1}$ with $\mu\left(F_{2} / F_{1}\right) \geq \mu\left(F_{1}\right)$. We conclude $\mu\left(F_{2}\right) \geq \mu\left(F_{1}\right)$, contradicting the choice of $F_{1}$. So $F / F_{1}=0$ and $F$ is semi-stable.

Suppose now $\delta(\vec{\omega})<0$. We know from the previous part that $F \in \boldsymbol{C}_{q}$ for some $q \in \mathbf{Q}$. We choose an exact sequence in $\boldsymbol{C}_{q}$

$$
\eta: 0-\rightarrow S-\rightarrow F-\rightarrow G-\rightarrow 0
$$

with $S \in C_{q}$ simple (= stable). If $G \neq 0, \eta$ does not split, so $\operatorname{Hom}(G, S(\vec{\omega})) \neq 0$. Hence $q=\mu(G) \leq \mu(S(\vec{\omega})<\mu(S)=q$, a contradiction.

\subsection{Theorem. Suppose $C$ has virtual genus one. Then}

(i) Each indecomposable vector bundle $F$ on $C$ is semi-stable.

(ii) Each $\boldsymbol{C}_{q}$ is closed under the formation of Auslander-Reiten sequences; in particu$\operatorname{lar} C_{q}(\vec{\omega})=C_{q}$.

(iii) Each $\boldsymbol{C}_{q}$ is a uniserial category. Accordingly ind $\left(\boldsymbol{C}_{q}\right)$ decomposes into AuslanderReiten components, which all are tubes of finite rank.

(iv) $\operatorname{Hom}(F, G) \neq 0$ for $F \in \boldsymbol{C}_{q}, G \in \boldsymbol{C}_{q^{\prime}}$ implies $q \leq q^{\prime}$ in $\mathbf{Q}$.

Actually it is possible to prove a stronger assertion in (iii), namely

$$
\boldsymbol{C}_{q}=\underset{x \in C}{\Perp} \boldsymbol{C}_{q, x}
$$

with uniserial categories $\boldsymbol{C}_{q, x}$ having $p(x)$ isomorphism classes of simple modules.

Proof: (i) is covered by Proposition 5.5.

(ii) Since $\delta(\vec{\omega})=0$, clearly $\boldsymbol{C}_{q}=\boldsymbol{C}_{q}(\vec{\omega})$ for each $q \in \mathbf{Q}$, so $\boldsymbol{C}_{q}$ is closed under Auslander-Reiten translation, hence under the formation of Auslander-Reiten sequences, since $C_{q}$ is closed under extensions.

(iii) Let $S, T$ be simple objects in $\boldsymbol{C}_{q}$. Since $\boldsymbol{C}_{q}$ is extension- closed in $\operatorname{coh}(\boldsymbol{C})$, calculation of extension of $S, T$ in $\boldsymbol{C}_{q}$ can be done in $\operatorname{coh}(\boldsymbol{C})$, so by Serre-duality

$$
\operatorname{Ext}^{1}(S, T)=\operatorname{Hom}(T, S(\vec{\omega})) \text {. }
$$

Hence $\operatorname{Ext}^{1}(S, T) \neq 0$ if and only if $T \tilde{=} S(\vec{\omega})$; moreover if $\operatorname{Ext}^{1}(S, T) \neq 0$ it has dimension 
one over $k$. By a classical result of representation theory [13], $\boldsymbol{C}_{q}$ is uniserial hence each $F \in C_{q}$ is uniquely determined by its simple socle $S$ and its length $n$. Notation: $F=S^{(n)}$. Since Auslander-Reiten translation $F \rightarrow F(\vec{\omega})$ is given by an automorphism of finite order (note $p \vec{\omega}=0$ ), all Auslander-Reiten components of $\boldsymbol{C}_{q}$ are actually tubes, whose rank, i.e. the number of isomorphisms classes of simple objects in $\boldsymbol{C}_{q}$, is a divisor of $p$.

(iv) is covered by Proposition 5.2.

5.7. Remark. Let first $\boldsymbol{C}$ denote a weighted projective line of arbitrary virtual genus. Since $\mathrm{Q} \operatorname{coh}(\boldsymbol{C})$ has global dimension one, each $X \in \mathrm{D}^{b}(\operatorname{coh}(\boldsymbol{C}))$ decomposes in $\mathrm{D}^{b}(\mathrm{Q} \operatorname{coh}(\boldsymbol{C}))$, hence in $\mathrm{D}^{b}(\operatorname{coh}(\boldsymbol{C}))$ into a (finite) direct sum of complexes $T^{n} A_{n}$ with $A_{n} \in \operatorname{coh}(\boldsymbol{C})$. Hence ind $\left(\mathrm{D}^{b}(\operatorname{coh}(\boldsymbol{C}))\right)$ can be calculated as the Ext - category of ind $(\operatorname{coh}(\boldsymbol{C}))$, whose objects are pairs $(\boldsymbol{F}, n)$ with $\boldsymbol{F} \in$ ind $(\operatorname{coh}(\boldsymbol{C})), n \in \mathbf{Z}$; morphisms are given by $\operatorname{Hom}((\boldsymbol{F}, n),(\boldsymbol{G}, m))=\operatorname{Ext}^{m-n}(\boldsymbol{F}, \boldsymbol{G})$, and composition is defined by means of the Yoneda composition.

If $g_{v}(\boldsymbol{C})=1$ and $\Lambda$ is the attached canonical algebra of tubular type. Theorem 5.6 allows easily to calculate the Auslander-Reiten quiver of $\operatorname{ind}\left(\mathrm{D}^{b}(\bmod \Lambda)\right)=\operatorname{ind}\left(\mathrm{D}^{b}(\operatorname{coh}(C))\right)$, as done by Happel and Ringel [22] by different methods.

5.8. Example. We are now going to sketch how the curves $C$ of virtual genus one arise as "quotients" $T / G$ of a smooth elliptic curve $T$ by a suitably chosen action of a finite algebraic group $G$. Here, we restrict to the case $(\boldsymbol{p}, \lambda)=(2,2,2,2 ; \lambda), \lambda \neq 0,1$.

We consider the plane elliptic curve $T \subset P_{2}(k)$ given by the equation $f=0$, where

$$
f=U_{0} U_{2}^{2}-U_{1}\left(U_{1}-U_{0}\right)\left(U_{1}-\lambda U_{0}\right) \text {. }
$$

The projective coordinate algebra

$$
A=k\left[U_{0}, U_{1}, U_{2}\right] /(f)
$$

of $T$ admits a $\mathbf{Z}_{2} \times \mathbf{Z}$-graduation given by

$$
\operatorname{deg}\left(U_{0}\right)=\operatorname{deg}\left(U_{1}\right)=(0,1), \operatorname{deg}\left(U_{2}\right)=(1,1),
$$

inducing a $\boldsymbol{\mu}_{2}$-action on $T$, where $\boldsymbol{\mu}_{2}$ is the algebraic group of second roots of unity. (On points of $T$ this action is given by $\left.\left[u_{0}, u_{1}, u_{2}\right]-\rightarrow\left[u_{0}, u_{1},-u_{2}\right]\right)$. If $\psi: T-\rightarrow T / \boldsymbol{\mu}_{2}=\boldsymbol{Y}$ denotes the quotient map, the direct image sheaf $\psi_{*}\left(\boldsymbol{O}_{\boldsymbol{T}}\right):=\boldsymbol{O}_{Y}$ carries a $\mathbf{Z}_{2}$-graduation corresponding to the $\boldsymbol{\mu}_{2}$-action on $T$. Contrary to the usual approach, where the zero-component of $\boldsymbol{O}_{Y}$ serves as the structure sheaf for $T / \boldsymbol{\mu}_{2}$, we define $\operatorname{coh}(\boldsymbol{Y})$ as the category of all coherent $\mathbf{Z}_{2}$-graded $\boldsymbol{O}_{Y}$ - modules. 
We are now going to prove that $\operatorname{coh}(\boldsymbol{Y}) \tilde{=} \operatorname{coh}(\boldsymbol{C}(2,2,2,2 ; \lambda))$, which justifies our assertion $T / \boldsymbol{\mu}_{2}=C$.

First, we note that the map $\phi: \mathbf{Z}_{2} \times \mathbf{Z}-\rightarrow \mathbf{L}(\boldsymbol{p})$, given on generators by $\phi(0,1)=3 \vec{x}_{0}$, $\phi(1,1)=\vec{x}_{1}+\vec{x}_{2}+\vec{x}_{3}$ allows to identify $\mathbf{Z}_{2} \times \mathbf{Z}$ with a subgroup of $\mathbf{L}(\boldsymbol{p})$. Moreover, with

$$
U_{0}=x_{0}^{3}, U_{1}=x_{0} x_{1}^{2}, U_{2}=x_{1} x_{2} x_{3}
$$

it is easy to see that the restriction $\underset{(u, n) \in \mathbf{Z}_{2} \times \mathbf{Z}}{\bigoplus_{\phi(u, n)}}$ of $S=S(\boldsymbol{p}, \lambda)$ to $\mathbf{Z}_{2} \times \mathbf{Z}$ is isomorphic to $A$ as a $\mathbf{Z}_{2} \times \mathbf{Z}$-graded algebra.

Moreover, one checks that $M \in \bmod ^{\mathrm{L}(p)}(S)$ has finite length if and only its restriction $\bar{M}=\underset{(u, n) \in \mathbf{Z}_{2} \times \mathbf{Z}}{\bigcup_{\phi(u, n)}} M_{\text {as }}$ finite length in $\bmod ^{\mathbf{Z}_{2} \times \mathbf{Z}}(A)$. Hence restriction $M \rightarrow \bar{M}$ induces an equivalence

$$
\bmod ^{\mathbf{L}(p)}(S) / \bmod _{0}^{\mathbf{L}}(\boldsymbol{p})(S)-\rightarrow \bmod ^{\mathbf{Z}_{2} \times \mathbf{Z}}(A) / \bmod _{0}^{\mathbf{Z}_{2} \times \mathbf{Z}}(A)
$$

of quotient categories. The category on the left is just $\operatorname{coh}(\boldsymbol{C}(\boldsymbol{p}, \lambda))$, by a variant of Serre's theorem the category on the right is equivalent to $\operatorname{coh}(\boldsymbol{Y})$.

\section{References}

1. M. F. Atiyah, "Vector bundles over an elliptic curve," Proc. London Math. Soc., 7, pp. 414-452 (1957).

2. M. Auslander and I. Reiten, "Representation theory of Artin algebras III," Comm.Algebra, 3, pp. 239-294 (1975).

3. M. Auslander, "Functors and morphisms determined by objects and applications of morphisms determined by modules," Proc. conf. representation theory, Philadelphia 1976, pp. 1-244, Marcel Dekker, New York (1978).

4. M. Auslander, "Rational singularities and almost split sequences," Trans. Amer. Math. Soc., 293, pp. 511 - 532 (1986).

5. A. A. Beilinson, "Coherent sheaves on $P_{n}$ and problems of linear algebra," Funct. Anal. Appl., 12, pp. 214-216 (1979).

6. M. Beltrametti and L. Robbiano, Introduction to the theory of weighted projective spaces, Preprint MPI Bonn (1985).

7. K. Bongartz, "Tilted algebras," Representations of algebras, pp. 26 - 38, Springer Verlag, Berlin - Heidelberg - New York (1981). Lecture Notes in Mathematics 903.

8. C. Delorme, "Espaces projectifs anisotropes," Bull. Soc. Math. France, 103, pp. 203-223 (1975). 
9. V. Dlab and C. M. Ringel, "Indecomposable representations of graphs and algebras," Mem. Amer. Math. Soc., 173 (1976).

10. I. Dolgachev, "Weighted projective varieties," Group actions and vector fields, pp. 34 - 71, Springer - Verlag, Berlin - Heidelberg - New York (1982). Lecture Notes in Mathematics 956.

11. P. Donovan and M. R. Freislich, The representation theory of finite graphs and associative algebras, Ottawa (1973). Carleton Lecture Notes 5.

12. P. Gabriel, "Des catégories abéliennes.," Bull. Soc. math. France, 90, pp. 323-448 (1967). [MR 38 \#1144 (T.-Y. Lam) = Zbl. 201, p. 356].

13. P. Gabriel, "Indecomposable representations II," Symposia Math. Ist. Naz. Alta Mat., 11, pp. 81-107 (1973).

14. P. Gabriel, "Auslander-Reiten sequences and representation- finite algebras," Proceedings Ottawa 1979, pp. 1-71, Springer-Verlag, Berlin-Heidelberg-New York (1980). Lecture Notes in Mathematics 831.

15. R. Godement, Théorie des faisceaux, Hermann, Paris (1973).

16. A. Grothendieck, "Sur la classification des fibrés holomorphes sur la surface de Riemann," Amer. J. Math., 79, pp. 121 - 138 (1957).

17. A. Grothendieck, Eléments de géométrie algébrique II, Publications Mathématiques 8, Institut des Hautes Etudes Scientifiques, Paris (1961).

18. A. Grothendieck, Eléments de géométrie algébrique III, Publications Mathématiques 11, Institut des Hautes Etudes Scientifiques, Paris (1961).

19. D. Happel, U. Preiser, and C. M. Ringel, "Binary polyhedral groups and Euclidean diagrams," Manuscripta math., 31, pp. 317-329 (1980).

20. D. Happel and C. M. Ringel, "Tilted Algebras," Trans. Amer. Math. Soc., 274, pp. 399-443 (1982).

21. D. Happel, "Triangulated categories in representation theory of finite dimensional algebras," Comm. Math. Helv. (1986). to appear.

22. D. Happel and C. M. Ringel, "The derived category of a tubular algebra," Representation theory I, Finite dimensional algebras, pp. 156 -180, Springer-Verlag, Berlin Heidelberg - New York (1986). Lecture Notes in Mathematics 1177.

23. R. Hartshorne, Residues and duality, Lecture Notes in Mathematics 20, Springer-Verlag, Berlin-Heidelberg-New York (1966). 
24. R. Hartshorne, Algebraic geometry, Graduate Texts in Mathematics 52, Springer-Verlag, Berlin-Heidelberg-New York (1977).

25. F. Klein, Vorlesungen über das Ikosaeder und die Auflösung der Gleichungen vom fünten Grade, Teubner, Leipzig (1884).

26. H. Lenzing, "Curve singularities arising from the representation theory of tame hereditary Artin algebras," Representation theory I. Finite dimensional Algebras, pp. 199-231, Springer-Verlag, Berlin-Heidelberg-New York (1986). Lecture Notes in Mathematics 1177.

27. S. Mori, “Graded factorial domains," Japan. J. Math., 3, pp. 223-238 (1977).

28. L. A. Nazarova, "Representations of quivers of infinite type," Izv. Akad. Nauk SSR, Ser. Math., 37, pp. 752 - 791 (1973).

29. T. Oda, "Vector bundles on an elliptic curve," Nagoya Math. J., 43, pp. 41-72 (1971).

30. C. M. Ringel, Tame algebras and integral quadratic forms, Springer, Berlin - Heidelberg - New York (1984). Lecture Notes in Mathematics 1099.

31. J.-P. Serre, "Faisceaux algébriques cohérents," Annals of Math., 61, pp. 197-278 (1955).

32. C. S. Seshadri, "Fibrés vectoriels sur les courbes algébriques," Astérisque, 96, pp. 1-209 (1982).

33. P. Slodowy, "Platonic solids, Kleinian singularities and Lie groups," Algebraic Geometry, pp. 102-138, Springer-Verlag, Heidelberg - New York (1983). Lecture Notes in Mathematics 1008.

34. J. L. Verdier, "Catégories dérivés, etat 0," Séminaire géométrie algébrique, 4 1/2, pp. 262-311, Springer-Verlag, Berlin - Heidelberg - New York (1977). Lecture Notes in Mathematics 569. 